\title{
The SDSS-IV Extended Baryon Oscillation Spectroscopic Survey: Baryon Acoustic Oscillations at Redshift of 0.72 with the DR14 Luminous Red Galaxy Sample
}

\author{
Julian E. Bautista $^{1,2}$ (D), Mariana Vargas-Magaña ${ }^{3}$, Kyle S. Dawson ${ }^{1}$ (D), Will J. Percival ${ }^{2}$, Jonathan Brinkmann ${ }^{4}$, Joel Brownstein ${ }^{1}$ (D), \\ Benjamin Camacho $^{3}$, Johan Comparat ${ }^{5}$, Hector Gil-Marín ${ }^{6,7}$, Eva-Maria Mueller ${ }^{3}$, Jeffrey A. Newman ${ }^{8}$ (D), Abhishek Prakash ${ }^{9}$ (D), \\ Ashley J. Ross ${ }^{10}$, Donald P. Schneider ${ }^{11,12}$, Hee-Jong Seo ${ }^{13}$, Jeremy Tinker $^{14}$, Rita Tojeiro ${ }^{15}$, Zhongxu Zhai ${ }^{14}$ (iD), and \\ Gong-Bo Zhao $2,16,17$ \\ 1 Department of Physics and Astronomy, University of Utah, Salt Lake City, UT 84112, USA; bautista@astro.utah.edu \\ 2 Institute of Cosmology \& Gravitation, Dennis Sciama Building, University of Portsmouth, Portsmouth, PO1 3FX, UK \\ 3 Instituto de Fisíca, Universidad Nacional Autonoma de Mexico, Apdo. Postal 20-364, 01000, D.F., Mexico \\ Apache Point Observatory, P.O. Box 59, Sunspot, NM 88349, USA \\ 5 Max-Planck-Institut für Extraterrestrische Physik, Giessenbachstraße, D-85748 Garching, Germany \\ 6 Sorbonne Universités, Institut Lagrange de Paris (ILP), 98 bis Boulevard Arago, F-75014 Paris, France \\ 7 LPNHE, CNRS/IN2P3, Université Pierre et Marie Curie Paris 6, Université Denis Diderot Paris 7, 4 place Jussieu, F-75252 Paris CEDEX, France \\ 8 Department of Physics and Astronomy and PITT PACC, University of Pittsburgh, Pittsburgh, PA 15260, USA \\ 9 Infrared Processing and Analysis Center, California Institute of Technology, MC 100-22, Pasadena, CA 91125, USA \\ ${ }^{10}$ Center for Cosmology and Astro-Particle Physics, Ohio State University, Columbus, OH 43210, USA \\ 11 Department of Astronomy and Astrophysics, 525 Davey Laboratory, The Pennsylvania State University, University Park, PA 16802, USA \\ 12 Institute for Gravitation and the Cosmos, The Pennsylvania State University, University Park, PA 16802, USA \\ 13 Department of Physics and Astronomy, Ohio University, 251B Clippinger Labs, Athens, OH 45701, USA \\ 14 Center for Cosmology and Particle Physics, Department of Physics, New York University, 4 Washington Place, New York, NY 10003, USA \\ School of Physics and Astronomy, University of St Andrews, St Andrews, KY16 9SS, UK \\ 16 National Astronomy Observatories, Chinese Academy of Science, Beijing, 100012, People's Republic of China \\ 17 College of Astronomy and Space Sciences, University of Chinese Academy of Sciences, Beijing 100049, People's Republic of China \\ Received 2017 December 19; revised 2018 June 17; accepted 2018 June 20; published 2018 August 14
}

\begin{abstract}
The extended Baryon Oscillation Spectroscopic Survey (eBOSS) Data Release 14 sample includes 80,118 luminous red galaxies (LRGs). By combining these galaxies with the high-redshift tail of the BOSS galaxy sample, we form a sample of LRGs at an effective redshift $z=0.72$, covering an effective volume of $0.9 \mathrm{Gpc}^{3}$. We account for spurious fluctuations caused by targeting and by redshift failures, which were validated on a set of mock catalogs. This analysis is sufficient to provide a $2.5 \%$ measurement of spherically averaged baryon acoustic oscillations $(\mathrm{BAO}), D_{V}(z=0.72)=2377_{-59}^{+61}\left(r_{d} / r_{d}\right.$,fid $) \mathrm{Mpc}$, at $2.8 \sigma$ of significance. Together with the recent quasar-based BAO measurement at $z=1.5$ and forthcoming emission line galaxy-based measurements, this measurement demonstrates that eBOSS is fulfilling its remit of extending the range of redshifts covered by such measurements, laying the groundwork for forthcoming surveys such as the Dark Energy Spectroscopic Survey and Euclid.
\end{abstract}

Key words: distance scale - large-scale structure of universe

\section{Introduction}

Over the last decade, the expansion history of the universe has been measured to percent-level precision using observations of the baryon acoustic oscillations (BAO; Peebles \& Yu 1970; Sunyaev \& Zeldovich 1970; Bond \& Efstathiou 1987) in the distribution of galaxies. Multiple measurements from an increasing number of surveys have provided robust support for the standard $\Lambda \mathrm{CDM}$ cosmological model. Key early surveys such as the Sloan Digital Sky Survey (SDSS; York et al. 2000) and the 2-degree Field Galaxy Redshift Survey (2dFGRS; Colless et al. 2003) generated spectroscopic samples for the BAO measurements given in Eisenstein et al. (2005), Percival et al. (2007) and Percival (2001), Cole et al. (2005), respectively. These spectroscopic programs were followed by the WiggleZ survey at higher redshift (Drinkwater et al. 2010) and the 6-degree Field Galaxy Survey (6dFGS; Jones et al. 2009) at lower redshift, also measuring BAO (Beutler et al. 2011; Blake et al. 2011, respectively). The Baryon Oscillation Spectroscopic Survey (BOSS; Dawson et al. 2013), conducted as part of the Sloan Digital Sky Survey III (SDSS-III; Eisenstein et al. 2011), provided the first BAO measurements with precision better than 1\% (Anderson et al. 2012, 2014a, 2014b; Tojeiro et al. 2014).
Results from the final sample of BOSS galaxies are presented in Alam et al. (2017), while results using the final sample of BOSS Ly $\alpha$ forests are presented in Bautista et al. (2017) and du Mas des Bourboux et al. (2017).

These BAO measurements are all broadly consistent with a $\Lambda \mathrm{CDM}$ cosmological model as inferred from Planck satellite measurements (Planck Collaboration I et al. 2016; Planck Collaboration XIII et al. 2016). Even so, the low value of the cosmological constant constrained by these data has as yet no compelling theoretical explanation (see Weinberg et al. 2013 for a review). The increasing precision of cosmological measurements has renewed interest in alternative models that predict similar behavior with a different mechanism causing cosmological acceleration (see Clifton et al. 2012 for a review). The combination of BAO measurements with measurements from redshift-space distortions (RSD), supernovae, weak lensing, and other low-redshift cosmological probes has therefore recently seen renewed focus, with many planned upcoming experiments. The Taipan survey will observe approximately 2 million galaxies over half the sky at $z<0.4$ (Cunha et al. 2017). At higher redshifts, the Dark Energy Spectroscopic Instrument (DESI; DESI Collaboration 2016a, 2016b), the 4MOST Cosmology 
Table 1

Cosmological Parameters Used in This Work

\begin{tabular}{lcc}
\hline \hline & Fiducial & Mocks \\
\hline$\Omega_{M} h^{2}$ & 0.1417 & 0.1421 \\
$=\Omega_{c} h^{2}$ & 0.1190 & 0.1196 \\
$+\Omega_{b} h^{2}$ & 0.0220 & 0.0225 \\
$+\Omega_{\nu} h^{2}$ & 0.0006 & 0 \\
$h$ & 0.676 & 0.7 \\
$N_{\nu}$ & 3 & 3 \\
$\sigma_{8}$ & 0.8 & 0.816 \\
$n_{s}$ & 0.97 & 0.97 \\
\hline$r_{d}[\mathrm{Mpc}]$ & 147.78 & 147.13 \\
$D_{H}(z=0.72) / r_{d}$ & 19.94 & 19.70 \\
$D_{M}(z=0.72) / r_{d}$ & 17.88 & 17.50 \\
$D_{V}(z=0.72) / r_{d}$ & 16.62 & 16.32 \\
\hline
\end{tabular}

Redshift Survey (de Jong et al. 2016), and the European Space Agency Euclid mission (Laureijs et al. 2011) will observe an order of magnitude more galaxies. The extended BOSS (eBOSS; Dawson et al. 2016), part of the SDSS-IV experiment (Blanton \& Bershady 2017), is the largest spectroscopic galaxy survey running at this time. eBOSS extends the redshift range beyond the BOSS galaxy sample, to redshifts that will be covered by future surveys. The main goal of eBOSS is to measure BAO distances at $1.6 \%$ precision level using $500 \mathrm{k}$ clustering quasars, $1.4 \%$ using Ly $\alpha$ forests, $2.2 \%$ with $200 \mathrm{k}$ emission line galaxies (ELGs), and $1 \%$ from $300 \mathrm{k}$ luminous red galaxies (LRGs). With these galaxy and quasar samples, eBOSS will produce the best measurements of the growth rate of structures from RSD: $2.8 \%$, $3.3 \%$, and $2.5 \%$ precision, respectively, from quasars, ELGs, and LRGs (Zhao et al. 2016).

In this paper, we present the first BAO measurement using the LRG sample observed by eBOSS, at an effective redshift $z=0.72$. This sample, described in Section 2, is designed to extend the CMASS sample from BOSS (Reid et al. 2016) to higher redshift. This sample of galaxies is supplemented by the final BOSS galaxy sample. In addition to the new data, the analysis methods in this work were improved from previous BOSS studies:

1. Spurious fluctuations caused by noncosmological variations in target density are modeled from multiple linear regression.

2. Enhanced characterization of "redshift failures," that is, spectra where the redshift is not measured with sufficient statistical significance, is utilized.

3. Corrections for targeting inhomogeneity and spectroscopic incompleteness are applied on the random catalog instead of up-weighting galaxies, thus reducing shot noise and systematic errors in the two-dimensional correlation function. A similar procedure was used in the determination of the selection function of the WiggleZ survey (Blake et al. 2010).

The structure of the paper is as follows. Section 2 describes our data and mock catalogs. Our treatment of photometric systematic errors and redshift failures is presented in Sections 3.1 and 3.2 and tested using mock catalogs. The model for BAO in the correlation function and the technique for reconstructing integrated bulk flows that degrade the BAO feature are presented in Section 4. Section 5 shows results on data and the main BAO measurement. Table 1 presents the cosmological models employed in our work.

\section{Data}

The sample of galaxies used in this work is mainly composed of LRGs observed spectroscopically during the first two years of eBOSS (Dawson et al. 2016), the cosmological component of the fourth generation of the SDSS-IV (Blanton \& Bershady 2017). In order to increase tracer density, we combine the eBOSS sample with the $z>0.6$ BOSS CMASS galaxies (Alam et al. 2017) trimmed to the area covered by eBOSS observations. The larger density is essential in order to obtain good performances of the reconstruction technique (Section 4.3) and better errors. By using only $z>0.6$ CMASS galaxies, our measurement is correlated with the higher redshift measurement of Alam et al. (2017) at $0.5<z<0.75$, but it is independent of the other two redshift bins $(0.2<z<0.5$ and $0.4<z<0.6)$. The final analysis of the eBOSS LRG sample will be done jointly with CMASS $(z>0.6)$ and the ELG sample $(0.7<z<1.1)$. The BOSS CMASS galaxies represent about one-third of the full sample used in this work. These data are found in the SDSS Data Release $14^{18}$ (Albofathi et al. 2018).

\subsection{Galaxy Sample and Redshift Estimators}

The eBOSS spectroscopic targets were selected from optical (SDSS DR13, SDSS Collaboration 2016) and infrared (from the Wide-field Infrared Survey Explorer, WISE; Wright et al. 2010) imaging data with infrared forced photometry applied over positions of SDSS sources (Lang et al. 2014). The LRG target selection is fully described in Prakash et al. (2016). The selection algorithm was informed by Prakash et al. (2015) and applied over the full BOSS imaging footprint, yielding about $60 \mathrm{deg}^{-2}$ LRG targets, of which $50 \mathrm{deg}^{-2}$ were observed spectroscopically. In short, the main (extinction-corrected) SDSS magnitude cuts of this sample are given by

$$
z<19.95 \quad 19.9<i<21.8
$$

which makes the eBOSS LRG galaxies a completely disjoint set (in magnitudes, not redshift) from CMASS galaxies (Eisenstein et al. 2011). Additional color cuts,

$$
r-i>0.98 \quad i-z>0.625 \quad r-W 1>2(r-i),
$$

yield, on average, higher-redshift galaxies than CMASS while avoiding star contamination. The selection was tested over $466 \mathrm{deg}^{2}$ covered during the Sloan Extended Quasar, ELG, and LRG Survey (SEQUELS). An overview of this pilot survey can be found in Dawson et al. (2016) and in the DR12 data release (Alam et al. 2015)

Spectra were obtained by the Sloan $2.5 \mathrm{~m}$ telescope at Apache Point Observatory, New Mexico (Gunn et al. 2006). Two multiobject spectrographs (Smee et al. 2013) simultaneously project 1000 spectra per exposure, including about 20 calibration stars and $\sim 80$ empty regions (for modeling sky subtraction). Spectra cover a wavelength range from 3600 to $10,000 \AA$ with a resolution $R=1500-2600$. Sets of 15 minute exposures were taken until a typical target with $g=21.2$ and

\footnotetext{
${ }^{18}$ sdss.org/dr14
} 

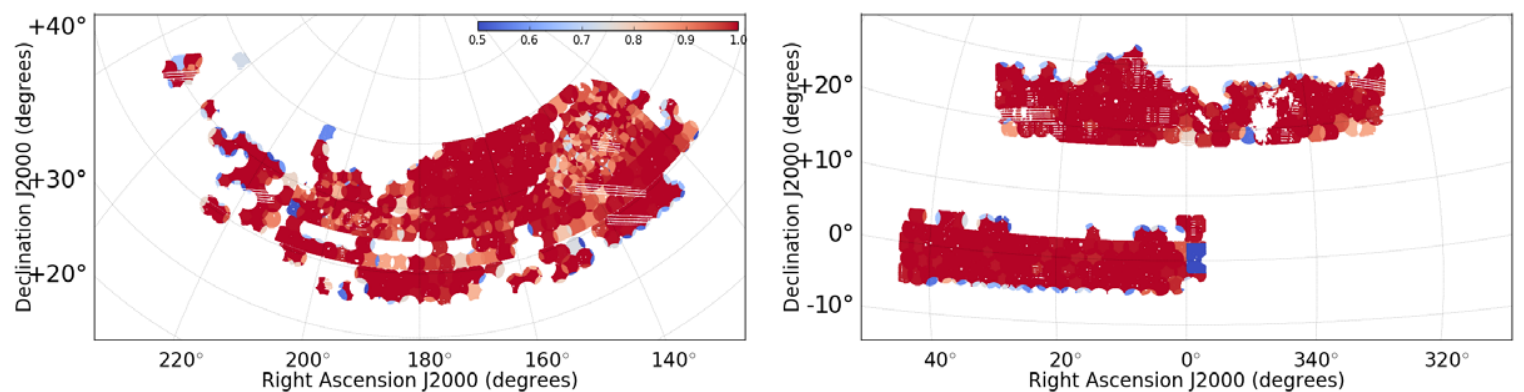

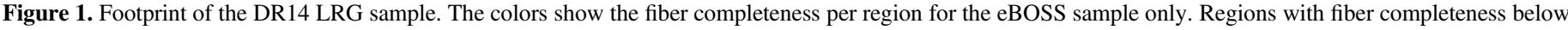
0.5 were removed from the final sample.

$i=20.2$ reaches a signal-to-noise ratio $(\mathrm{S} / \mathrm{N})$ of 3.16 per pixel in the $g$ band and 4.7 per pixel in the $i$ band.

Spectra were extracted, sky-subtracted, flux-calibrated, and coadded using version $\mathrm{V} 5 \_10 \_0$ of the software idlspec2d. ${ }^{19}$ The extraction algorithm has improved since BOSS; its description can be found in Appendix B of Bautista et al. (2017). Recent improvements on coaddition and fluxcalibration are described in Hutchinson et al. (2016) and Jensen et al. (2016). The final eBOSS LRG spectra were classified and their redshifts measured primarily by redmonster ${ }^{20}$ (Hutchinson et al. 2016), complemented by redshifts obtained using spec1d (Bolton et al. 2012). On average, $10 \%$ of the eBOSS LRG sample lacks a statistically confident redshift estimate due mainly to the low $\mathrm{S} / \mathrm{N}$ of their spectra. In Section 3.2 we discuss how this failure rate depends on many characteristics of the observations, such as position of the fiber in the focal plane or the position of the trace in the CCD. In that section, we present the methods used to account for these fluctuations in our clustering measurement.

\subsection{Catalog Creation}

One important step of the clustering analysis is to determine the survey mask. Usually the mask is defined by a set of random points that are Monte Carlo sampling the volume covered by the survey, referred to as the "random catalog" or simply "the randoms." This random catalog also accounts for angular variations in spectroscopic completeness of the survey. We follow here a procedure similar to that used for the final BOSS galaxy clustering measurements, described in Reid et al. (2016).

Starting from the photometric target sample, we first veto objects in "bad" photometric regions of the sky that were included in the target selection process. We exclude regions around stars in the Tycho catalog (Høg et al. 2000) with Tycho $B_{T}$ magnitudes within $[6,11.5]$ with a magnitude-dependent radius ranging from $3 ! 4$ to $0 ! 8$. An additional mask excludes regions $0.1-1.5$ in radius around bright galaxies and other objects (Rykoff et al. 2014). Bright objects in WISE imaging are also masked. Regions of radius from 16 ' 6 to $2^{\prime}$ are masked around sources with $W 1$ magnitudes ranging from 2 to 8 . We removed regions where galactic extinction $E(B-V)>0.15$ or where the seeing is larger than $2 ! 3,2 ! 1$, and $2 ! 0$ in the $g, r$, and $i$ bands, respectively. Bad photometric regions and bright objects mask $4.5 \%$ of the targets in the NGC and $12.1 \%$ of targets in the SGC. Since the eBOSS quasars and several other target classes have priority during the fiber assignment

\footnotetext{
19 Available at https://www.sdss.org/dr14/algorithms/software/products/.

${ }^{20}$ https://github.com/timahutchinson/redmonster/
}

procedure (Dawson et al. 2016), we are unable to obtain spectra from LRG targets that lie less than 62" (corresponding angular diameter of a fiber in the sky) from a higher priority target. This results in $7.7 \%$ of LRG targets being masked by quasar fibers in the NGC and $7.0 \%$ in the SGC. We expect that the effect on the LRG clustering caused by these knockouts is negligible given that other samples are relatively sparse and have little overlap in redshift with the galaxy sample. We leave tests on this assumption to future work. We mask a few tens of the remaining LRG targets that lie in the center of the focal plane, where a center post holds the plate and prevents fibers from being assigned within the $92^{\prime \prime}$ central radius. The total masked area is $12.3 \%$ for the NGC and $18.2 \%$ for the SGC. Targets that are masked in this process do not count in the fiber completeness calculations (see below).

The spectroscopic sample is then matched to the remaining targets. A small fraction of targets do not receive an optical fiber and therefore have no spectra or redshift information. Some of these missing redshifts are caused by the impracticality of placing two optical fibers on LRG targets closer than $62^{\prime \prime}$. We refer to these as fiber collisions. These collisions impact the small-scale clustering since they preferably occur in overdense regions. Different methods to correct for the effect of collisions have been studied in the past (e.g., Guo et al. 2012; Bianchi \& Percival 2017). For our catalogs, we simply upweight by one the target with redshift information, as performed in Alam et al. (2017). The impact on correlations larger than $10 h^{-1} \mathrm{Mpc}$ is negligible (Guo et al. 2012). Other targets do not receive a fiber because of the limited number of fibers available per observation (1000 fibers).

We correct for these missing targets by downsampling the random catalog so it follows the computed completeness. We define fiber assignment completeness as

$$
C=\frac{N_{\mathrm{gal}}+N_{\mathrm{qso}}+N_{\mathrm{star}}+N_{\mathrm{cp}}+N_{\mathrm{fail}}}{N_{\mathrm{targ}}},
$$

where $N_{\text {gal }}$ is the number of confirmed LRGs with redshifts, $N_{\text {qso }}$ and $N_{\text {star }}$ are the numbers of quasars and stars found among LRG targets (incorrect target classes), $N_{\mathrm{cp}}$ is the number of LRG targets without spectra due to a collision with a galaxy with a redshift (they count as being observed), and $N_{\text {fail }}$ is the number of targets with spectra but without a confident redshift (we correct for redshift failures in a later process; see Section 3.2). The fiber completeness is computed per "sector," where a sector is a connected region of the sky defined by a unique set of plates. We exclude sectors where the fiber completeness is below $50 \%$ to avoid regions covered by 
Table 2

Survey Specification of the eBOSS LRG Sample Used in This Analysis

\begin{tabular}{|c|c|c|c|c|c|c|c|c|}
\hline Survey & Cap & $N_{\text {gal }}$ & $N_{\text {zfail }}$ & $N_{\mathrm{cp}}$ & $N_{\mathrm{qso}}$ & $N_{\text {star }}$ & $A_{\text {eff }}\left[\mathrm{deg}^{2}\right]$ & $V_{\text {eff }}\left[\mathrm{Gpc}^{3}\right]$ \\
\hline eBOSS & NGC & 45826 & 4957 & 2263 & 18 & 2897 & 1033.4 & 0.356 \\
\hline \multirow[t]{2}{*}{$(0.6<z<1.0)$} & SGC & 34292 & 4366 & 1687 & 18 & 4273 & 811.6 & 0.263 \\
\hline & Total & 80118 & 9323 & 3950 & 36 & 7170 & 1845.0 & 0.619 \\
\hline CMASS & NGC & 26149 & & & & & 1033.4 & 0.174 \\
\hline \multirow[t]{2}{*}{$(0.6<z<1.0)$} & SGC & 20290 & & & & & 811.6 & 0.138 \\
\hline & Total & 46439 & & & & & 1845.0 & 0.312 \\
\hline eBOSS + & NGC & 71975 & & & & & 1033.4 & 0.513 \\
\hline CMASS & SGC & 54582 & & & & & 811.6 & 0.387 \\
\hline$(0.6<z<1.0)$ & Total & 126557 & & & & & 1845.0 & 0.900 \\
\hline
\end{tabular}

Note. Note that CMASS effective volume is computed using eBOSS effective area.

multiple plates, where unfinished observations potentially introduce an artificial pattern of clustering.

Figure 1 displays the footprint of the DR14 LRG sample where the color-coding indicates the corresponding fiber completeness. The top of Table 2 presents the number of LRGs, the total effective area (weighted by completeness) of our sample, and the effective volume (defined below) of our samples.

Using the fiducial cosmology presented in Table 1, we compute weights that optimize clustering signal-to-noise ratio for a survey with varying density as a function of redshift. Also known as FKP weights (Feldman et al. 1994), we apply a weight to each object,

$$
w_{\mathrm{FKP}}=\frac{1}{1+\bar{n}(z) P_{0}},
$$

where $\bar{n}(z)$ is the average comoving density of galaxies as a function of redshift and $P_{0}$ is the value of the power spectrum at scales relevant for our study $\left(k \sim 0.14 h \mathrm{Mpc}^{-1}\right.$; Font-Ribera et al. 2014). For the eBOSS LRG sample, we adopt a value of $P_{0}=10^{4} h^{-3} \mathrm{Mpc}^{3}$, which is the same value used in the final BOSS CMASS clustering measurements. The effective volume is defined as

$$
V_{\mathrm{eff}}=\int_{z=0.6}^{z=1.0}\left(\frac{\bar{n}(z) P_{0}}{1+\bar{n}(z) P_{0}}\right)^{2} A_{\mathrm{eff}} R^{2}(z) d R(z),
$$

where $R(z)$ is the comoving distance to redshift $z$ and $A_{\text {eff }}$ is the effective area (in steradians) of the survey.

We built the random catalog using a sample 50 times that of the galaxy sample size. We applied the same veto masks as for the observed targets. Redshifts are assigned to each random point so as to match the redshift distribution $\bar{n}(z)$ of the data. Figure 2 shows the redshift distribution of our samples, separately for the NGC and the SGC. We restrict our analysis to $z>0.6$ to avoid a larger overlap with the CMASS sample while not reducing the effective redshift. The cut at $z<1.0$ was chosen to avoid a low number density of LRGs. In Section 3, we describe how systematic effects caused by target selection and redshift failures are corrected using the same random catalog.

\subsection{Mock Catalogs}

We created a set of a thousand mock catalogs, each reproducing the angular and redshift distribution of galaxies in the DR14 sample (Figure 1), as well as the large-scale correlation function predicted from the fiducial cosmology. We produced simulations for both eBOSS and for the combined CMASS+eBOSS sample using redshift distributions shown in Figure 2.

Mock catalogs were created with the quick particle mesh (QPM) method (White et al. 2014), also used in recent clustering studies (Alam et al. 2017; Ata et al. 2018). Each realization consists of a different set of second-order Lagrangian perturbation theory (2LPT) initial conditions computed at $z=25$. These perturbations were evolved to $z=0.7$ using a low-force and low-mass resolution particle-mesh $\mathrm{N}$-body simulation, with time steps of $15 \%$ in the log of the scale factor. The runs employed here were based on a $2560 \mathrm{~h}^{-1} \mathrm{Mpc}$ side box containing $1280^{3}$ dark-matter particles. Halos were defined using a friends-of-friends algorithm with a linking length of $20 \%$ of the mean interparticle spacing. These halos are populated with galaxies following a halo occupation distribution (HOD) model derived from the small-scale clustering of the same LRG sample (Zhai et al. 2017). The best-fit HOD model yields a bias value of 2.3 for this sample, a satellite fraction of $13 \%$, and a mean halo mass of $2.5 \times 10^{13} h^{-1} M_{\odot}$. We subsample galaxies in order to reproduce the redshift distribution $n(z)$ and the angular fiber completeness as measured from the data. We introduce redshift failures into our mock catalogs by sampling from the model derived in Section 3.2.

\section{Correcting Noncosmological Density Fluctuations}

As described in the previous section, eBOSS LRG targets were selected to be strictly fainter in $i$-band magnitudes than the CMASS sample. Larger photometric errors in this regime create a higher rate of contamination by stars and, for some galaxy spectra, scatter from faint galaxies into the selection. Therefore, the eBOSS LRGs are more susceptible to contamination by inhomogeneities in target selection and by patterns in redshift failures than the CMASS galaxies from BOSS. In this section we introduce methods to account for this contamination. All work in this section is focused on the eBOSS sample only.

\subsection{Systematics Due to Photometry}

In BOSS clustering studies, it was found that galaxy density is correlated with stellar density and seeing (Ross et al. 2011, 2012, 2017; Ho et al. 2012). These correlations 


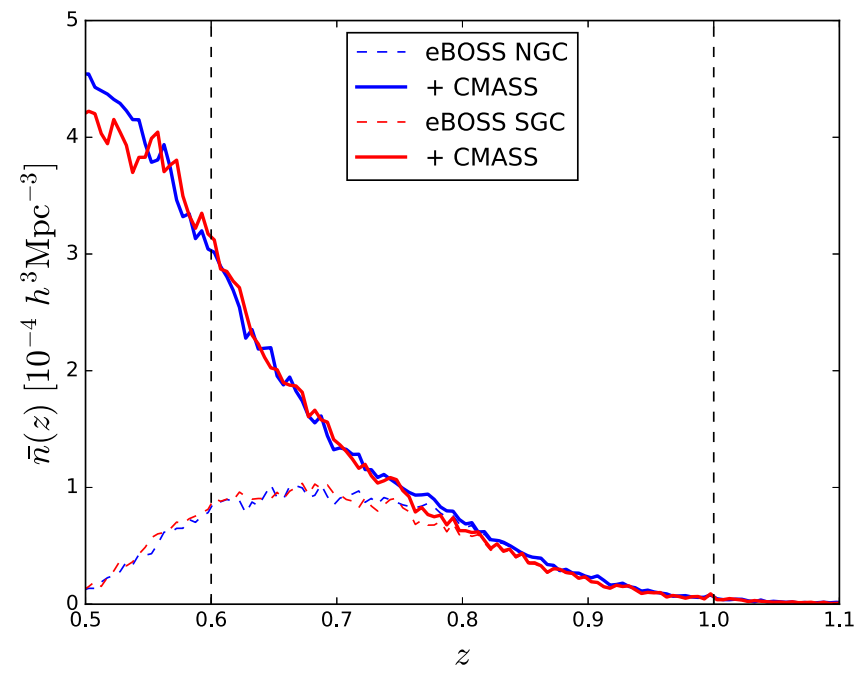

Figure 2. Density of LRGs as a function of redshift. Dashed vertical lines indicate the redshift range used in our clustering measurement. Here we see that a significant fraction of eBOSS LRGs have redshifts below 0.6, where CMASS are more numerous. We can remark on the importance of the CMASS sample between redshifts 0.6 and 0.7 .

contaminate our clustering measurements by introducing largescale power not associated with the true distribution of galaxies. In Ross et al. (2017), systematic weights were computed for each galaxy in order to counteract these dependencies, but assuming different systematics are independent. In this work, we drop the assumption of independent systematics and use a multiple linear regression, similar to that used to assess variations in the target selection of LRGs (Prakash et al. 2016), quasars (Myers et al. 2015), and ELGs (Delubac et al. 2017; Raichoor et al. 2017). The multiple linear regression has the advantage of automatically accounting for correlated systematics, such as stellar density and Galactic extinction. In performing the regression, we include only galaxies with confident spectroscopic redshifts in the region of interest $0.6<z<1.0$. The NGC and SGC samples are analyzed independently.

The multiple linear regression calculates a "density" model, $\delta_{\text {phot, }}$ as a linear combination of maps $m_{i}$ :

$$
\delta_{\text {phot }}(\text { R.A., decl. })=p_{0}+\sum_{i=1}^{n} p_{i} m_{i} \text { (R.A., decl.) }
$$

where $p_{0}$ is the average density over the full footprint, and $p_{i}$ (with $i>0$ ) are fitted coefficients that minimize $\chi^{2}=$ $\left(\delta-\delta_{\text {phot }}\right)^{2} / \sigma_{\delta}^{2}$. Each map $m_{i}$ is produced using Healpix with pixels of equal area of $189 \operatorname{arcmin}^{2}$. The observed fluctuations $\delta$ are estimated from the data (normalized ratio of number of galaxies and randoms) as a function of a given systematic value. The numbers of galaxies and randoms in each bin are weighted by $w_{\text {FKP }}$ (Equation (4)) in order to account for the cosmological fluctuations. Error bars are assumed to be Poisson on the weighted number of galaxies per bin.

Figure 3 shows the result of the regression using seven different maps. Five of these maps are derived from SDSS photometry (Doi et al. 2010; Aihara et al. 2011): stellar density, $i$-band depth, $z$-band sky flux, $z$-FWHM and $r$-band extinction, while two maps are derived from WISE photometry (Wright et al. 2010; Lang et al. 2014): median number of single-exposure frames per pixel in the WISE W1 band (WISE W1 Cov Med) and median of accumulated flux per pixel in the WISE $W 1$ band (WISE W1 Med). Since different SDSS bands (Fukugita et al. 1996) are strongly correlated, we restrict our analysis to a single band per systematic. In the NGC, $\chi^{2} /$ dof $=192.2 /(70-8)$ before corrections and $80.6 /(70-8)$ after corrections, while in the SGC we obtain $\chi^{2} /$ dof $=365.8 /(70-8)$ before corrections and $68.2 /(70-8)$ after corrections. The most important improvements are related to dependencies with stellar density, extinction, and WISE quantities. This analysis can be performed in many redshift bins, but no further improvement was obtained. Hereafter, we compute systematic maps using a single redshift bin.

Once the density model is derived, our sample can, in principle, be corrected either by applying to the galaxies a set of systematic weights defined as $w_{\text {sys }}=1 / \delta_{\text {phot }}$, or by subsampling the random catalog as a function of R.A. and decl. to mimic the density model.

Figure 4 presents the monopole and the quadrupole of the correlation function calculated using the standard Landy \& Szalay (1993) estimator. We show the observed correlation function before accounting for noncosmological fluctuations in target density and compare the results of correcting with an upweighting scheme to results from subsampling of randoms. Correlations are biased positive even for large separations where no cosmological signal is expected. As we can see in the figure, the quadrupole is barely affected by this kind of systematic error.

Differences between the two correction techniques are smaller than error bars and are consistent with being caused by the relatively smaller number of randoms for the subsampling case. Hereafter, targeting systematics are corrected by subsampling of randoms.

\subsection{Correcting for Redshift Failures}

Variations in the quality of spectroscopy can have a similar effect on clustering as variations in the quality of the photometry used to identify targets. Figure 5 reveals that lower-S/N spectra yield, on average, fewer statistically confident redshifts. We define redshift efficiency as

$$
\eta=\frac{N_{\mathrm{gal}}}{N_{\mathrm{zfail}}+N_{\mathrm{gal}}}
$$

where quantities are defined in Section 2.2. If the expected distribution of failures is not uniformly distributed across the sky, our clustering measurements might be biased. In this section, we introduce a new technique to account for these failures. Using mock catalogs (described in Section 2.3), we demonstrate that our method leads to unbiased clustering measurements.

In previous studies from BOSS, redshift failures were accounted for by an up-weighting technique. These spectra lacking a confident redshift transfer their weight to the nearest object in the sky with a confident redshift (either a galaxy or a star). In other words, the nearest neighbor of a failure will count double when counting pairs. This simple correction procedure leads to unbiased results on scales far larger than the average separation between a failure and a nonfailure and as long as the rate of redshift failures is low. For example, in the BOSS CMASS sample, the overall failure rate was $1.8 \%$ and the median separation was 3.8 arcmin. In the eBOSS LRG sample, 

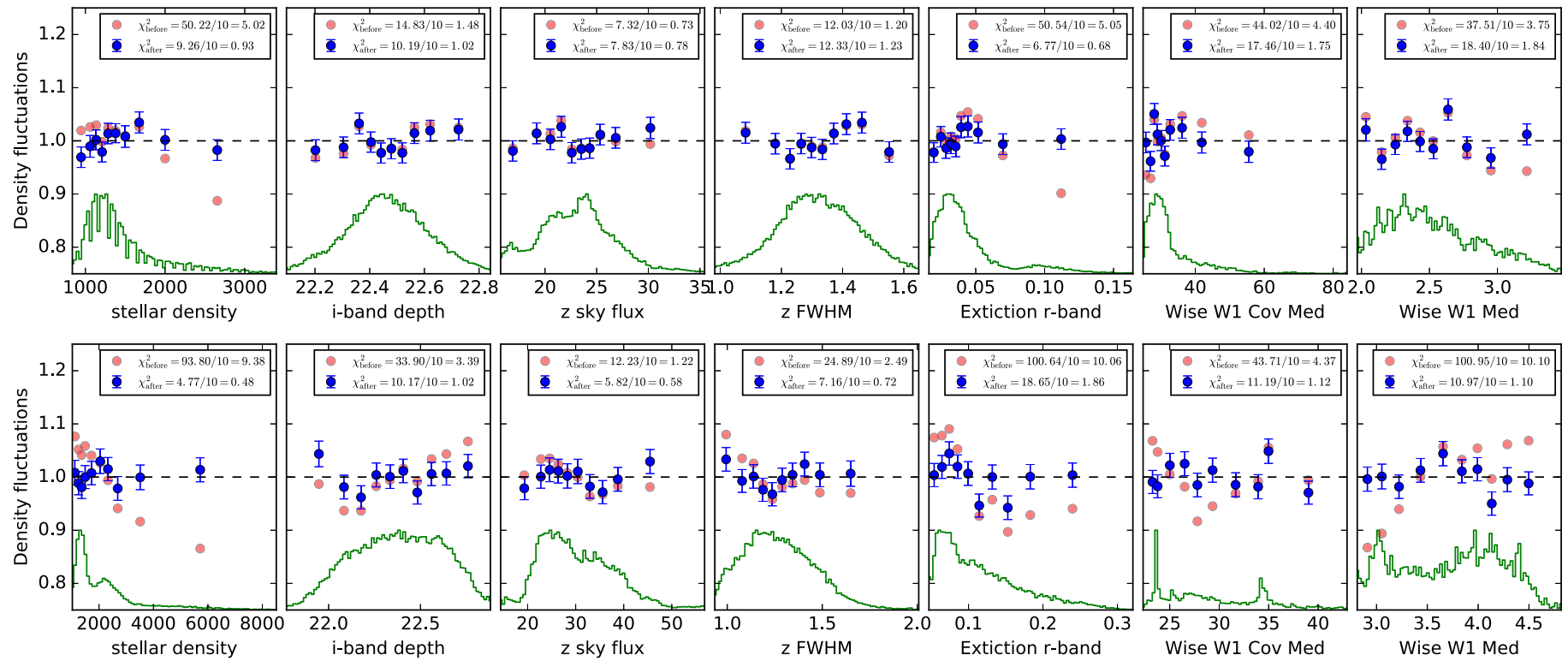

Figure 3. Fluctuations of galaxy number density with respect to different photometric quantities for the NGC (top panels) and the SGC (bottom panels). Red (blue) points indicate fluctuations before (after) corrections. The distribution of the same quantities among galaxies is shown in green. The fit is made simultaneously across all maps, accounting automatically for correlations between different maps.
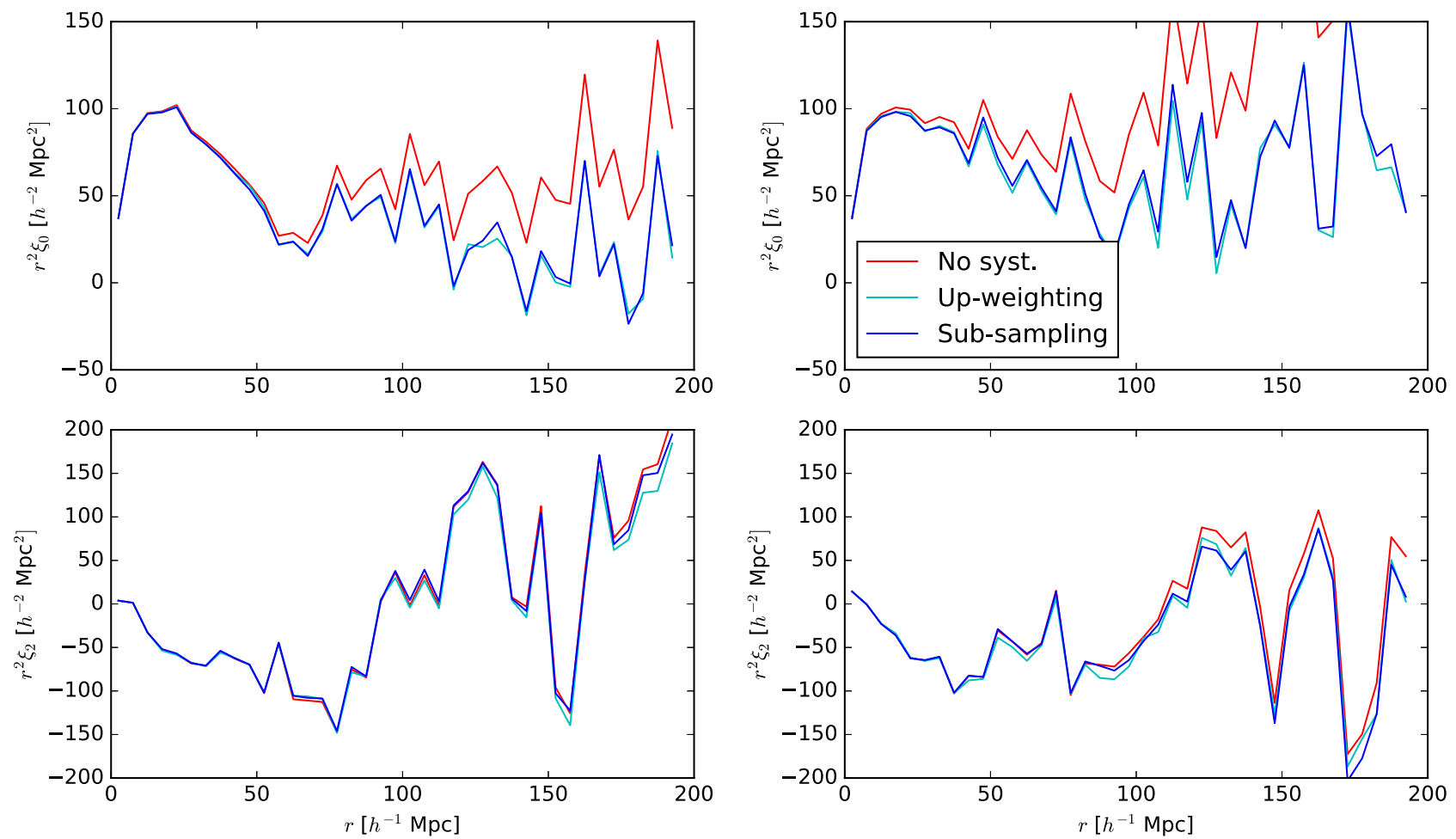

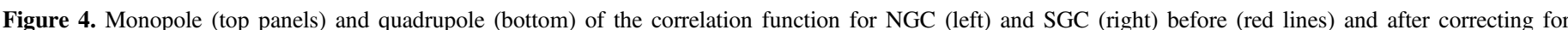

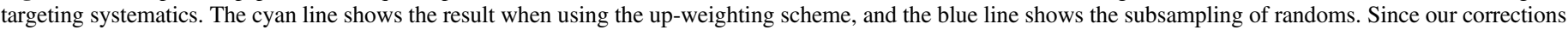

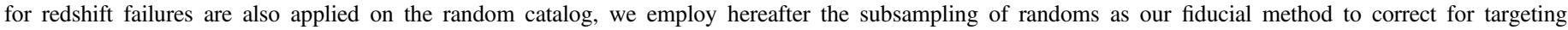
systematics.

the failure rate is around $10 \%$ and the median separation is 5 arcmin. The higher failure rate and larger average separation force us to revisit the manner in which redshift incompleteness is addressed in eBOSS.

Instead of using the up-weighting technique, we derive a model describing the probability of an observation of a target galaxy yielding a confident redshift. In our model, this probability depends on the position of its fiber in the focal plane and the overall $\mathrm{S} / \mathrm{N}$ of the plate. The model for failures is then applied to the random sample, by subsampling, mimicking the patterns retrieved in our model.

Figure 6 shows the probability of obtaining a confident redshift (hereafter called the redshift efficiency) for a galaxy as a function of its position in the focal plane. We observe a decrease in this probability near the side edges of the focal plane. The reason for this behavior is that the light transmitted 


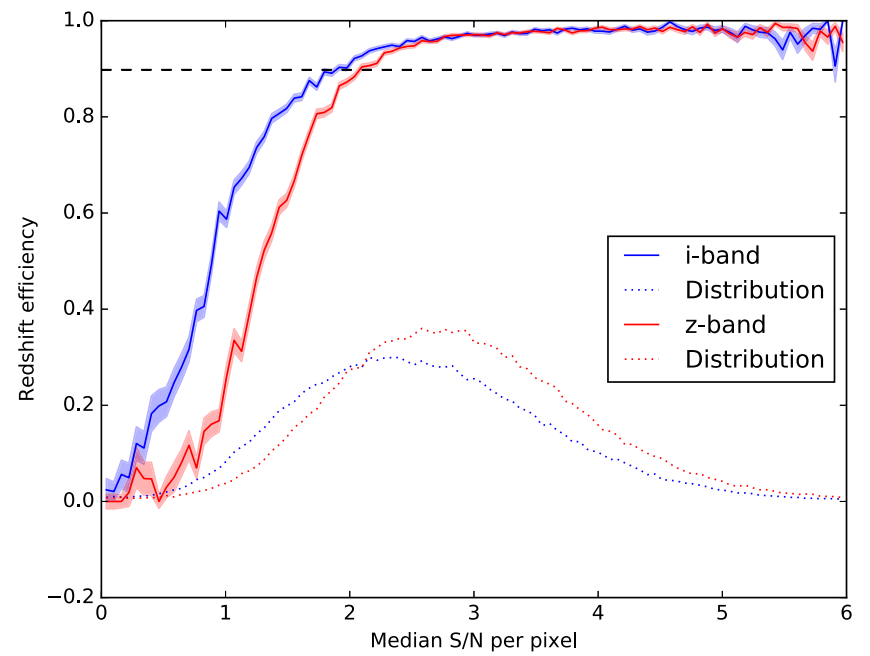

Figure 5. Average redshift efficiency as a function of median pixel $\mathrm{S} / \mathrm{N}$ in the $i$ (blue line) and $z$ (red line) SDSS bands. Dotted lines indicate the distribution of median $\mathrm{S} / \mathrm{N}$ of the eBOSS LRG sample. The dashed line represents the average redshift efficiency of the whole sample of $90.5 \%$.

through fibers near the side edges of the focal plane arrive near the edges of the CCD, where the optical performance inside the spectrographs is degraded, leading to a larger point-spread function and optical aberrations such as coma (Smee et al. 2013). By associating each random with a plate and location within the plate, we can use the binned probabilities to subsample the random catalog. We divide the focal plane into 20 bins across the diameter of the focal plane in Cartesian coordinates, XFOCAL and YFOCAL, that range from -326 to $326 \mathrm{~mm}$. Our choice of bin size is large enough to minimize Poisson noise in galaxy counts but small enough to identify the failure-rate pattern on the scales of interest.

Figure 7 presents this dependency of the redshift efficiency with $\mathrm{S} / \mathrm{N}$ of both spectrographs for each plate. We use these halfplates since two independent spectrographs have different throughput (Smee et al. 2013). On average, fibers lying on the YFOCAL $<0$ region (in Figure 6) encounter spectrograph \#1, while the others are typically imaged through spectrograph \#2. Independent measurements of $\mathrm{S} / \mathrm{N}$ are made per spectrograph and per optical band (corresponding to SDSS $g, r, i$ ). We used $i$-band values only, given that most of the signal of LRG spectra is observed in the $i$ band. The binned data (blue points) are included in order to reduce Poisson noise. We fit the efficiency $\eta$ using a simple model

$$
\eta(x)=\frac{1}{1+1 / p(x)},
$$

where $x=(\mathrm{S} / \mathrm{N})^{2}$, and $p(x)$ is a first-order polynomial. This choice of model ensures the correct asymptotic behavior when $\mathrm{S} / \mathrm{N} \rightarrow 0$ or $\mathrm{S} / \mathrm{N} \rightarrow \infty$. The best-fit models yield $\chi^{2} /$ dof $=$ $47.1 /(20-2)$ for the NGC and $\chi^{2} /$ dof $=39.2 /(20-2)$ for the SGC. These values are higher than unity, indicating that efficiencies may depend on factors other than spectrograph $\mathrm{S} / \mathrm{N}$. However, we were not able to identify any other significant factors. We expect to improve the model of redshift failures with a larger data sample.

Our final efficiency model is the product of the two efficiencies given in Figure 6 and Equation (8). We normalize the efficiencies such that the final product is consistent with the average spectrograph efficiency given by the red line in Figure 7 (since the latter already includes the average focal plane efficiencies).

The subsampling technique is implemented as follows. For each random galaxy, we assign a plate XFOCAL and YFOCAL values based on its location in the sky. In overlap regions covered by more than a single plate, a random plate value among those observing this region is assigned to this random galaxy. Given the plate number and the (R.A., decl.) of each random, we can assign a position in the focal plane of that plate. We draw random numbers, and we remove random galaxies based on the probability given by the model.

In order to test our procedure, we included redshift failures on the set of 1000 mock catalogs for eBOSS galaxies, following the model derived from real data. We first assign mock galaxies to plates (and their XFOCAL and YFOCAL), as done with the randoms. For each galaxy, we randomly convert it as a failure based on the probability given by the model. We apply two methods to account for these failures: the nearestneighbor up-weighting and the subsampling of randoms. For the subsampling case, we use redshift failures from the mock itself to derive individual redshift efficiency models, employing the same algorithm that is used to derive the inefficiency model from the data. Doing so accounts for the Poisson noise that could be caused by the binned data.

Figure 8 shows the impact of different correction methods on the average correlation function of the 1000 mock catalogs. The reference correlation function is computed from the same mock catalogs without any synthetic redshift failures. The gray region represents the error on the mean of 1000 correlation functions (all curves have similar errors). The nearest-neighbor up-weighting scheme (red curve) introduces structure into the monopole with amplitude smaller than the error on the 1000 mocks, except at scales below $20 h^{-1} \mathrm{Mpc}$, which are usually discarded in BAO analyses. However, this scheme introduces a bias of at least $5 \%$ on all scales in the amplitude of the quadrupole. This bias is likely caused by the larger fraction of failures and lower densities of objects compared to previous measurements that used this technique (e.g., CMASS), effectively affecting larger scales. We created mocks with twice the rate of failures, yielding a bias of about $10 \%$ in the quadrupole. This indicates that the bias introduced by this correction technique might be proportional to the failure rate of the sample. The subsampling technique (blue lines) has better performance than up-weighting for all scales, for both monopole and quadrupole, yielding no significant bias at this level of precision. We also applied two "noncomplete" versions of the subsampling scheme, where we assume the efficiency model depends only on one factor: either focal plane position or only spectrograph $\mathrm{S} / \mathrm{N}$. Even when our assumed model for redshift failures is not complete, our model is superior to the up-weighting scheme.

The bias on the quadrupole introduced by up-weighting would yield biased estimates of the growth rate of structures in studies of RSD. We recommend that future work on RSD using galaxy samples containing significant failure rates implement this correction scheme. A similar correction procedure was used on the WiggleZ survey (Blake et al. 2010).

We use the subsampling techniques on the eBOSS LRG sample in all clustering measurements in this work. For the CMASS $z>0.6$ sample, we employ the weights used in the 

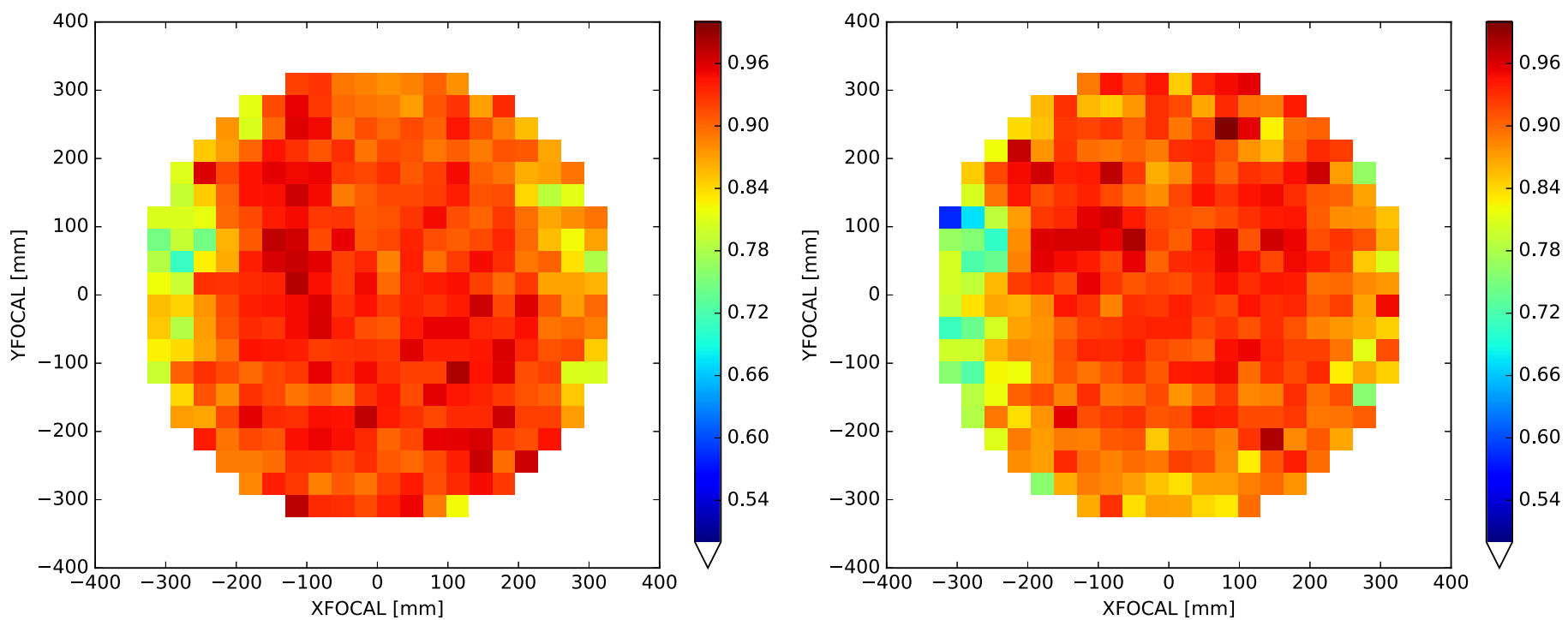

Figure 6. Average redshift efficiency as a function of physical position of the optical fiber in the focal plane. The left (right) panel shows the NGC (SGC) failure rates. The region where YFOCAL is negative corresponds to spectrograph \#1, while YFOCAL $>0$ corresponds to spectrograph \#2.
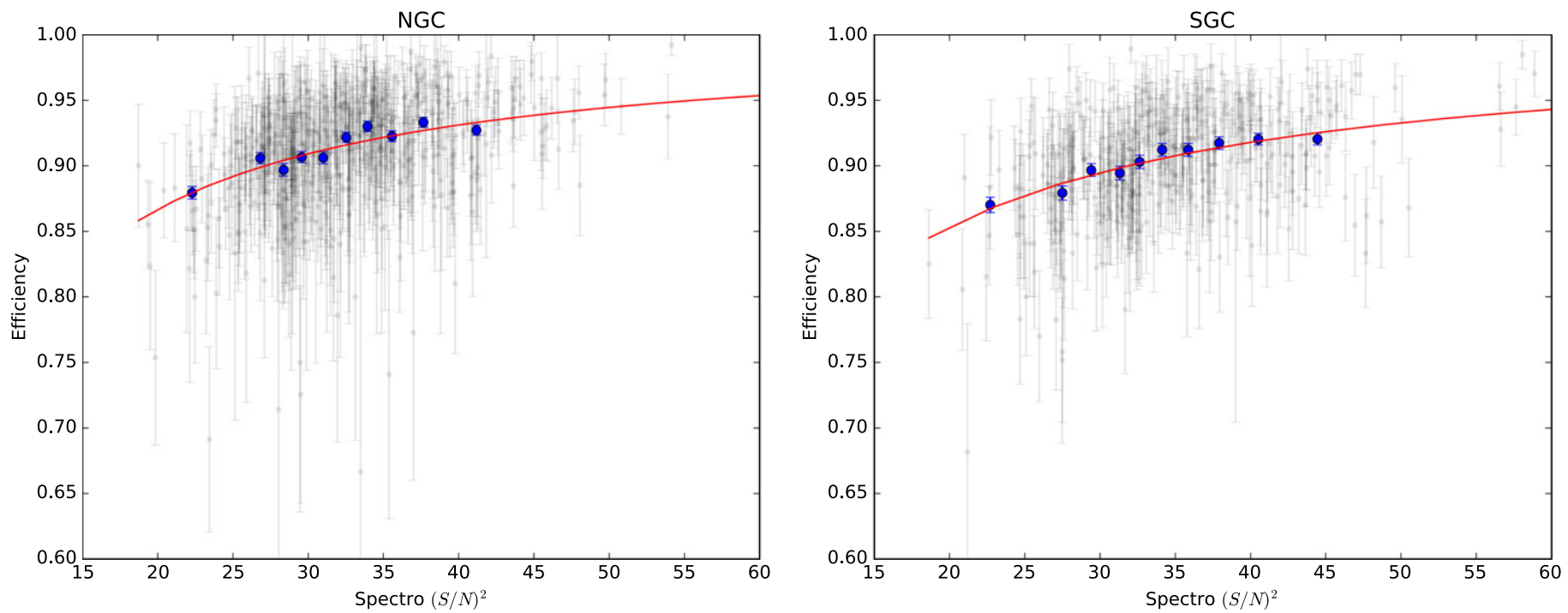

Figure 7. Average redshift efficiency per spectrograph as a function of its $\mathrm{S} / \mathrm{N}$ squared binned (blue points) and unbinned (gray points). Red lines show the best-fit models. The left panel shows the results for the NGC, while the right panel shows the SGC. S/N is computed as the median per pixel over the $i$-band wavelength range.

final BOSS measurements, which use the up-weighting technique. We combine CMASS and eBOSS samples, both data and randoms, making sure that the ratio of number of galaxies over randoms is the same.

\section{The Model and Fitting Methodology}

In this section, we describe the model used to fit the correlation function, the reconstruction procedure applied, and tests on the mock catalogs.

\subsection{The Model}

To fit the measured correlation function, we follow the standard approach described in previous papers (e.g., Alam et al. 2017; Ata et al. 2018). The model redshift-space correlation function is obtained from a Fourier transform of the power spectrum, which is defined as

$$
\begin{aligned}
P(k, \mu)= & \frac{b^{2}\left[1+\beta\left(1-e^{-k^{2} \Sigma_{r}^{2} / 2}\right) \mu^{2}\right]^{2}}{\left(1+k^{2} \mu^{2} \Sigma_{s}^{2}\right)} \\
& \times\left[P_{\operatorname{lin}}(k)+P_{\text {peak }}(k)\left(e^{-k^{2} \Sigma_{\mathrm{nl}}^{2}(\mu)}-1\right)\right],
\end{aligned}
$$

where $b$ is the linear bias, $\beta$ is the RSD parameter, $k$ is the modulus of the wave vector, and $\mu$ is the cosine of the angle between the wave vector and the line of sight. We introduce anisotropic nonlinear broadening of the BAO peak by multiplying the "peak-only" power spectrum $P_{\text {peak }}$ by a Gaussian term with $\Sigma_{\mathrm{nl}}^{2}(\mu)=\Sigma_{\|}^{2} \mu^{2}+\Sigma_{\perp}^{2}\left(1-\mu^{2}\right)$. The nonlinear random motions on small scales are modeled by a Lorentzian term parameterized by $\Sigma_{s}$. All fits have $\left(\Sigma_{\|}, \Sigma_{\perp}, \Sigma_{s}\right)$ values fixed. The values for these damping terms were computed by fitting the average of many mocks: $\left(\Sigma_{\|}, \Sigma_{\perp}, \Sigma_{s}\right)=(9.5,6.0$, 

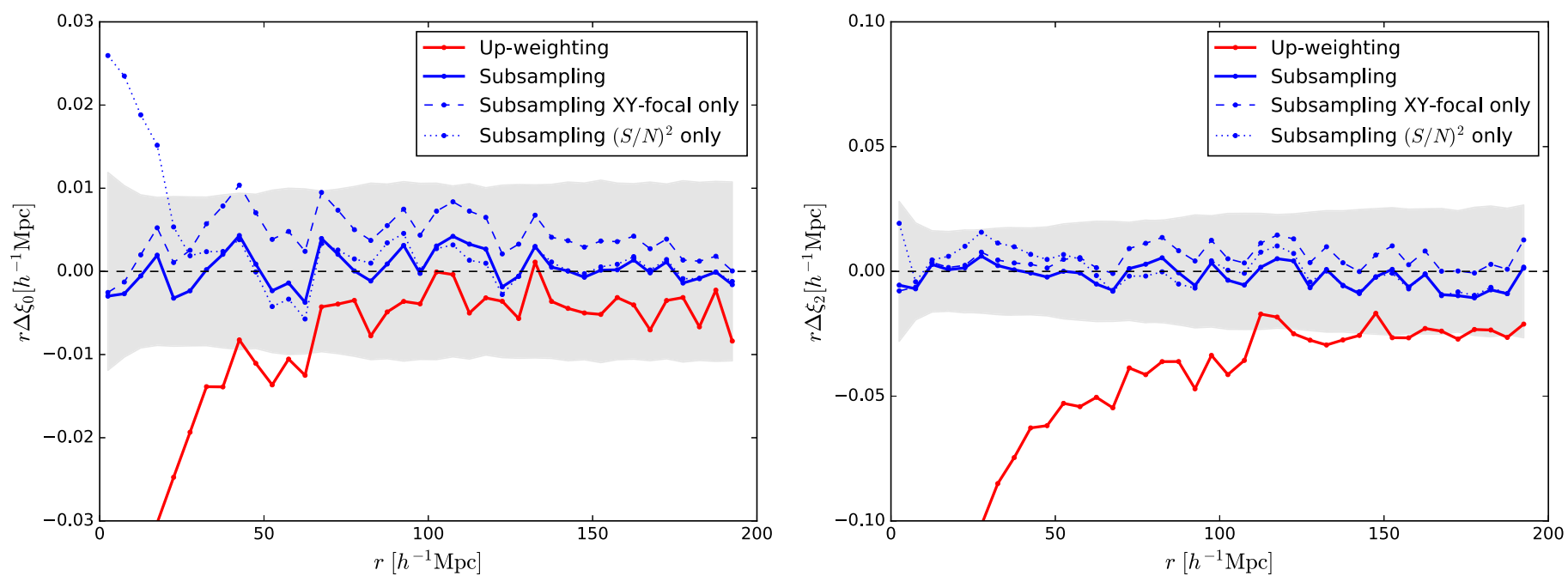

Figure 8. Difference between the average mock correlation function where redshift failures are corrected and the mocks without synthetic failures. The left panel presents the monopole and the right panel shows the quadrupole, both scaled by $r$ for clarity. Different lines present the results with different schemes. The gray band is the error on the mean of 1000 mock catalogs of the eBOSS NGC sample (without CMASS). The up-weighting of the nearest-neighbor technique introduces a bias of $5 \%$ in the quadrupole on all scales, while the subsampling is unbiased.

2.0) $h^{-1} \mathrm{Mpc}$ for prereconstruction and $\left(\Sigma_{\|}, \Sigma_{\perp}, \Sigma_{s}\right)=(5.5$, $5.5,0) h^{-1} \mathrm{Mpc}$ for postreconstruction. Results are insensitive to this choice (for values not too far from these). Following the theoretical motivation of White (2015) and Seo et al. (2016), we apply a term $S(k)=e^{-k^{2} \Sigma_{r}^{2} / 2}$ to the postreconstruction modeling of the correlation function. This term models the smoothing used in our reconstruction technique, where $\Sigma_{r}=15 h^{-1} \mathrm{Mpc}$ (see Section 4.3). This term was used in the BOSS DR12 results of Ross et al. (2017) and Beutler et al. (2017). We follow Kirkby et al. (2013) to compute $P_{\text {peak }}$ from the linear power spectrum $P_{\text {lin }}$, by computing its correlation function, fitting a third-order polynomial function over the peak region, and transforming back to Fourier space. The linear power spectrum $P_{\text {lin }}$ is computed using the code $\mathrm{CAMB}^{21}$ (Lewis et al. 2000) with cosmological parameters of our fiducial cosmology (Table 1).

In practice, we derive multipoles for the correlation function $\xi_{\ell}(r)$ from multipoles of the power spectrum $P_{\ell}(k)$, simply defined as

$$
P_{\ell}(k)=\frac{2 \ell+1}{2} \int_{-1}^{1} P(k, \mu) L_{\ell}(\mu) d \mu
$$

where $L_{\ell}$ are Legendre polynomials. The correlation function is

$$
\xi_{\ell}(r)=\frac{i^{\ell}}{2 \pi^{2}} \int_{0}^{\infty} k^{2} j_{\ell}(k r) P_{\ell}(k) d k
$$

where $j_{\ell}$ are the spherical Bessel functions.

In order to measure the BAO peak position, we scale separations $r$ with an isotropic dilation factor, $\alpha_{\text {iso }}$, defined as the ratio of the "spherically averaged" distance

$$
D_{V}(z)=\left[z D_{M}^{2}(z) D_{H}(z)\right]^{1 / 3}
$$

\footnotetext{
${ }^{21}$ camb.info
}

to the sound horizon scale $r_{d}$, normalized to its value in our fiducial cosmology:

$$
\alpha_{\text {iso }}=\frac{D_{V}\left(z_{\mathrm{eff}}\right) / r_{d}}{D_{V}^{\text {fid }}\left(z_{\mathrm{eff}}\right) / r_{d}^{\text {fid }}} .
$$

Another choice of parameterization of the BAO peak position uses two dilation parameters that decompose the scaling into transverse, $\alpha_{\perp}$, and radial, $\alpha_{\|}$, directions. These quantities are related, respectively, to the comoving angular diameter distance, $D_{M}=(1+z) D_{A}(z)$, and to the Hubble distance, $D_{H}=c / H(z)$, by

$$
\alpha_{\perp}=\frac{D_{M}\left(z_{\mathrm{eff}}\right) / r_{d}}{D_{M}^{\text {fid }}\left(z_{\mathrm{eff}}\right) / r_{d}^{\text {fid }}} \quad \quad \alpha_{\|}=\frac{D_{H}\left(z_{\mathrm{eff}}\right) / r_{d}}{D_{H}^{\text {fid }}\left(z_{\mathrm{eff}}\right) / r_{d}^{\text {fid }}}
$$

In our implementation, we apply the scaling factors in the twodimensional power spectrum (Equation (9)) before computing its multipoles and the associated Hankel transforms (e.g., Beutler et al. 2014; Gil-Marín et al. 2017).

Unknown systematic effects in the survey might create largescale correlations that could contaminate our measurements. Also, there is no accurate model for the postreconstruction correlation function to date (the term on $\Sigma_{r}$ is not sufficient). We take into account any spurious correlations or mismodeling by introducing an additive, smooth function of separation. Our final template can be written as

$$
\xi_{\ell}^{t}(r)=\xi_{\ell}(\alpha r)+a_{\ell 0}+\frac{a_{\ell 1}}{r}+\frac{a_{\ell 2}}{r^{2}} .
$$

For isotropic fits, we only fit the monopole of the correlation function, fixing the value of $\beta=0.3$. For anisotropic fits, we fit both monopole and quadrupole, leaving $\beta$ as a free parameter and fitting $b$ with a flat prior between $b=1.0$ and 3 . For all fits, the broadband parameters are free, while dilation parameters are fitted in the range $0.5<\alpha<1.5$. A total of five parameters are fitted on isotropic fits and 10 parameters for anisotropic fits.

\subsection{Parameter Estimation}

The best model is found by minimizing $\chi^{2}=d \hat{W} d^{T}$, where $d$ is the monopole (and quadrupole) of the correlation function, 
and $\hat{W}$ is the estimated precision matrix, defined as the inverse of the estimated covariance matrix $\hat{C}$. We use the iMinuit python package $^{22}$ that implements a quasi-Newton method using the Davidon-Fletcher-Powell (DFP) formula to find minima. All of our covariance matrices are derived from the scatter of measurements from mock catalogs. In order to account for the finite number of mock measurements used to derive $\hat{C}$, the unbiased estimator for the precision matrix (Hartlap et al. 2007; Percival et al. 2014; Taylor et al. 2014) should be written as

$$
\hat{W}=\left(1-\frac{n}{N_{\text {mocks }}-1}\right) \hat{C}^{-1},
$$

where $n$ is the number of elements in the data vector $d$.

Errors from best-fit parameters are derived from the $\Delta \chi^{2}=1$ region of the marginalized $\chi^{2}$ profiles. Errors on the BAO scale $\alpha_{\text {iso }}$ are usually asymmetric for the current $\mathrm{S} / \mathrm{N}$ of our samples.

\subsection{Reconstruction}

To reduce the nonlinear effects on the BAO feature, we applied "reconstruction" (Eisenstein et al. 2007; Burden et al. 2015; Vargas-Magaña et al. 2017) to the eBOSS +CMASS sample. The reconstruction method reverses a fraction of nonlinear motion of the overdensities, sharpening the BAO peak and thus increasing the precision of our distance-redshift measurement.

Using the Zeldovich approximation, we calculate Lagrangian displacements $\boldsymbol{\Psi}$ based on an estimate of the velocities made from the density field. In order to estimate the galaxy density field, we insert the NGC or the SGC region inside a box with width 3.6 comoving $h^{-1} \mathrm{Gpc}$, assigning the galaxies and randoms into a grid of $512^{3}$ cells (using our fiducial cosmology). We use the cloud-in-cell scheme to assign galaxies and randoms to the grid. ${ }^{23}$ The density is smoothed using a three-dimensional Gaussian kernel with $15 h^{-1} \mathrm{Mpc}$ as its characteristic length. Burden et al. (2014) and VargasMagaña et al. (2017) studied how results vary as a function of this smoothing length and found that $15 h^{-1} \mathrm{Mpc}$ is close to optimal in terms of sharpening the BAO peak for densities similar to those matching our samples.

For a redshift-space density field, an approximate solution for the displacement field using inverse fast Fourier transforms (IFFT) can be written as (Burden et al. 2015)

$$
\boldsymbol{\Psi}=\operatorname{IFFT}\left[\frac{-i \boldsymbol{k} \tilde{\delta}(\boldsymbol{k})}{b k^{2}}\right]-\frac{f}{b+f}\left(\operatorname{IFFT}\left[\frac{-i \boldsymbol{k} \tilde{\delta}(\boldsymbol{k})}{b k^{2}}\right] \cdot \hat{r}\right) \hat{r},
$$

where $\tilde{\delta}$ is the Fourier transform of the density field, $\boldsymbol{k}$ is the wave vector, $b$ and $f$ are values for the bias and growth rate for the sample, and $\hat{r}$ is the unit vector in the radial direction. Because the overdensity field in redshift space is not irrotational, this equation is only an approximation, although it can be made more accurate, tending toward the true solution, using an iterative approach (Burden et al. 2015).

Once the displacement field has been calculated, we move both data and randoms by the expected value to obtain the reconstructed catalogs. We can also remove RSD by moving

\footnotetext{
22 http://iminuit.readthedocs.io

23 A discussion on the effects of assignment scheme on fast Fourier transforms can be found in Cui et al. (2008).
}

galaxies by an additional displacement defined as

$$
\boldsymbol{\Psi}_{\mathrm{RSD}}=-f(\boldsymbol{\Psi} \cdot \hat{r}) \hat{r} .
$$

We consider three implementations of the reconstruction method. The differences between them are whether or not we remove RSD and how many iterations we perform, as in Burden et al. (2015):

A. Not removing RSD and not performing iterations

B. Removing RSD and not performing iterations

C. Removing RSD and performing iterations

For case B, we use Equation (19) in Burden et al. (2015).

\subsection{Fitting Mock Catalogs}

We computed the correlation function for all 1000 mock catalogs of the eBOSS+CMASS samples, pre- and postreconstruction. Our fiducial cosmology was employed to compute comoving separations. The monopoles and quadrupoles for the mock catalogs are displayed in Figure 9 and compared with the data. For scales of interest for BAO $\left(r>30 h^{-1} \mathrm{Mpc}\right)$, the mock and data results mostly agree, except on a few points in the quadrupole where the data show deviations of about $3 \sigma$ from the mean of the mocks. The source of this deviation was not identified, but broadband terms (Equation (15)) in our fits are able to marginalize over part of these deviations.

We performed fits of the BAO scale over $30<r<$ $180 h^{-1} \mathrm{Mpc}$ on the set of 1000 mock catalogs in order to check for any possible biases or misestimation of errors. Table 3 and Figure 10 show results for isotropic fits, while Table 4 and Figure 11 present anisotropic results. Dilation factors are compared to their expected values, which are not unity given the different cosmological models used in the simulations and the analysis (see Table 1).

For the isotropic fits, values of $\alpha_{\text {iso }}$ are consistent with the input within the $2 \sigma$ level (where $\sigma$ is the error on the average of 1000 mocks) for two of the reconstruction methods (B and C). The rms of $\alpha_{\text {iso }}$ values are slightly larger than the average permock estimated error due to outliers of $\alpha_{\text {iso. The average gain }}$ in estimated errors caused by reconstruction is $\sim 20 \%$ and is clearly visible in Figure 10. The significance of BAO detections is estimated in individual mocks by comparing the $\chi^{2}$ of a model with a peak to the $\chi_{\text {no peak }}^{2}$ of a model without a peak: $\Delta \chi^{2}=\chi_{\text {no peak }}^{2}-\chi^{2}$. Prereconstruction mocks show $\left\langle\Delta \chi^{2}\right\rangle=10.1$, corresponding to $\sim 3.2 \sigma$ detection. Reconstruction slightly increases the value of $\Delta \chi^{2}$, from 10.1 to above 11.0. The average significance reaches $3.4 \sigma$ for case $C_{\text {. Less }}$ than $6 \%$ of mocks produce no BAO detection, where $\Delta \chi^{2}<1$, even after reconstruction is applied.

Anisotropic fits on mock catalogs yield slightly worse results than isotropic fits due to the current low statistical power of this sample. Figure 11 reveals slightly thinner distributions of $\alpha_{\perp}$ and $\alpha_{\|}$after applying reconstruction. However, the mean values of $\alpha$ show a small bias relative to the expected input values. These biases represent $20 \%$ of the expected error of a single realization for prereconstruction mocks, and they are reduced to $5 \%-15 \%$ in postreconstruction cases. These biases are caused mostly by the low statistical power of the current LRG sample, which makes the $\alpha$ distributions non-Gaussian. If we fit the average correlation function of 1000 mock catalogs, we are able to recover the input values within error bars. The average $\Delta \chi^{2}$ is increased by up to two units in postreconstruction cases. Since we have two BAO 

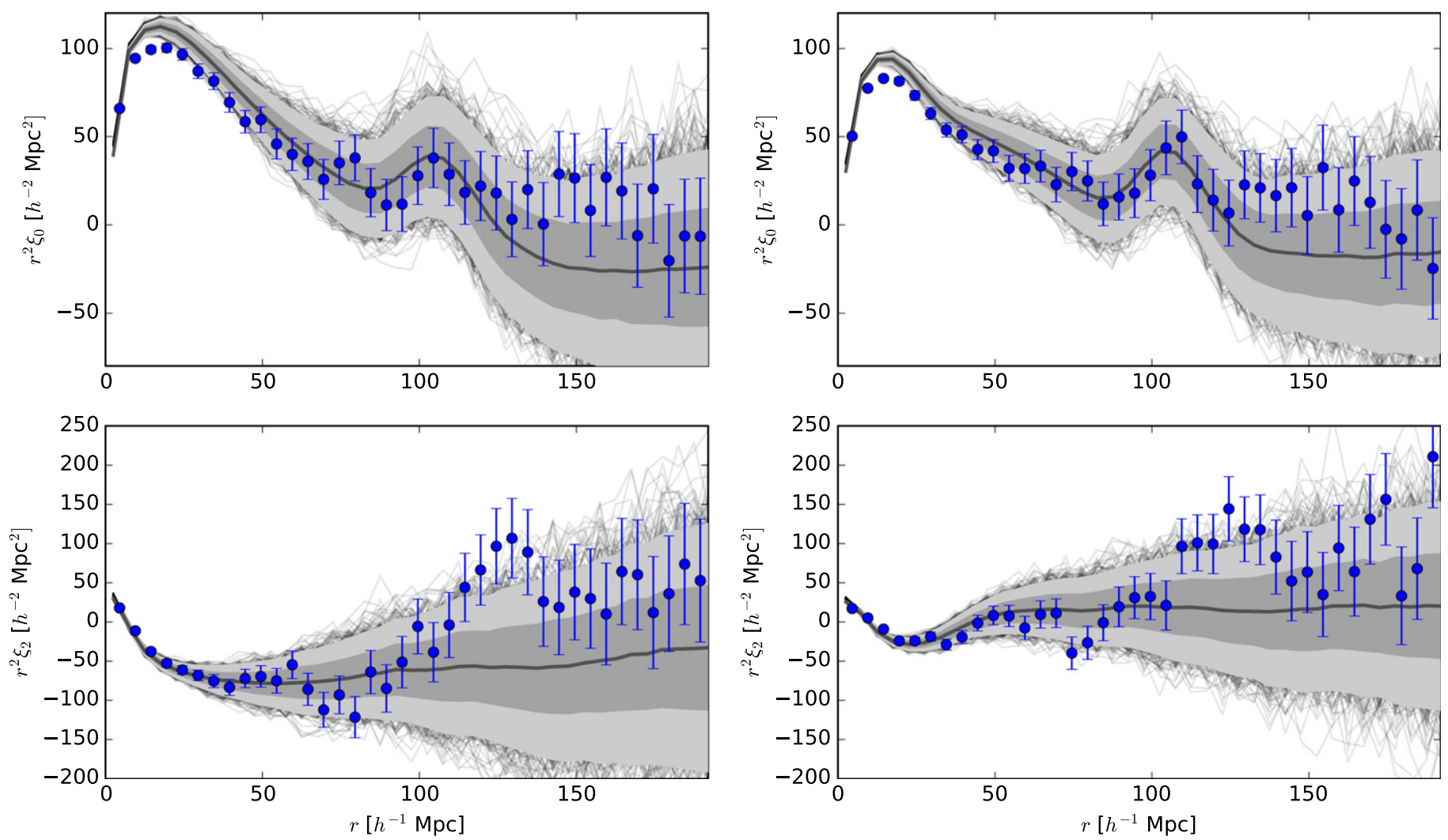

Figure 9. Comparison of the monopole (top panels) and quadrupole (bottom panels) of data (blue points) and mock catalogs (gray lines) of the eBOSS+CMASS combined NGC+SGC. Panels on the left (right) show the pre(post)reconstruction catalogs. The shaded regions represent the $68 \%$ and $95 \%$ boundaries of the distributions of correlation functions around the mean. Mocks and data correlation functions agree on most scales, except in the monopole at $r<30 h^{-1}$ Mpc (excluded from our analysis) and around $r \sim 120 h^{-1} \mathrm{Mpc}$ in the quadrupole.
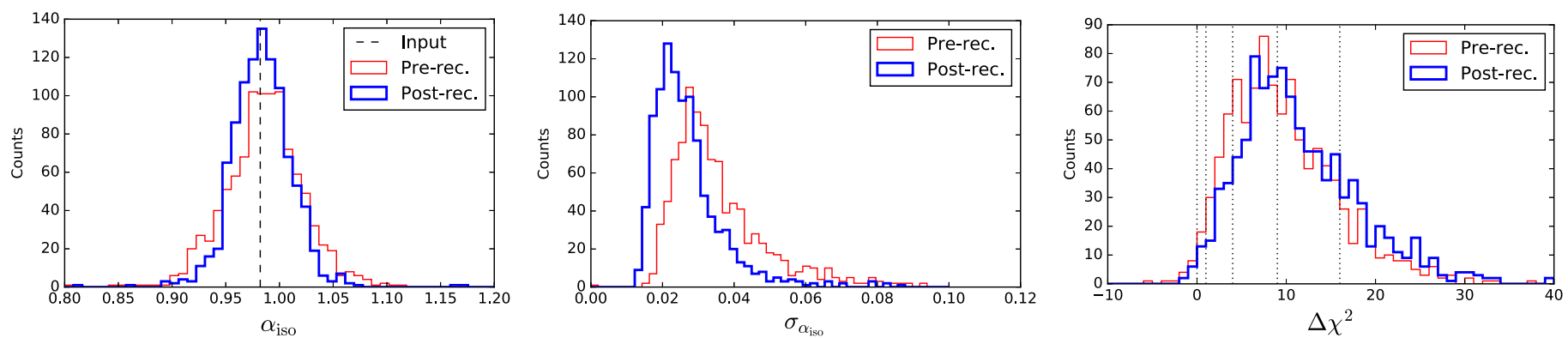

Figure 10. Distribution of best-fit values for $\alpha_{\text {iso }}$ (left), estimated errors (center), and the $\Delta \chi^{2}$ (right panel) for the set of 1000 mock catalogs. Results before reconstruction are presented in red, while results after reconstruction (method C) are presented in blue. Dashed lines in the left panels indicate the expected value given the cosmological model in Table 1. Dotted lines in the right panel indicate $\Delta \chi^{2}=\{0,1.0,4.0,9.0,16.0\}$, corresponding to BAO detection significances of $0,1,2,3$, and $4 \sigma$, respectively.

Table 3

Results of Isotropic Fits on the Full Set of 1000 Mock Catalogs of eBOSS+CMASS NGC+SGC

\begin{tabular}{lccccc}
\hline \hline Case & $N_{\text {good }}$ & $\left\langle\alpha_{\text {iso }}-\alpha_{\text {iso } 0}\right\rangle$ & rms $\left(\alpha_{\text {iso }}\right)$ & $\left\langle\sigma_{\alpha_{\text {iso }}}\right\rangle$ & $\left\langle\chi_{r}^{2}\right\rangle$ \\
\hline Pre-rec. & 965 & $0.0041 \pm 0.0012$ & 0.037 & 0.036 & 0.97 \\
Post-rec (A) & 972 & $0.0066 \pm 0.0009$ & 0.028 & 0.028 & 0.96 \\
Post-rec (B) & 958 & $0.0012 \pm 0.0010$ & 0.030 & 0.028 & 0.96 \\
Post-rec (C) & 979 & $0.0015 \pm 0.0009$ & 0.027 & 0.026 & 11.0 \\
\hline
\end{tabular}

Note. Given the cosmological parameters in Table 1, the input value of $\alpha_{\text {isoo }}$ is 0.9821 . All fits use $\Delta r=5 h^{-1} \mathrm{Mpc}$ bins. The column $N_{\text {good }}$ shows the number of mocks where $\Delta \chi^{2}>1.0$ from which all numbers are computed.

parameters instead of one, the corresponding significance postreconstruction is $\sim 3 \sigma$. If mock catalogs are a good representation of real data, we expect a $\sim 4.6 \%$ measurement on $\alpha_{\perp}$ and $\sim 8.4 \%$ on $\alpha_{\|}$postreconstruction.
All three reconstruction procedures show similar performances when applied to mock catalogs. On isotropic fits, methods $\mathrm{B}$ and $\mathrm{C}$ have smaller biases on $\alpha_{\text {iso }}$ and slightly larger significance on BAO detection than 

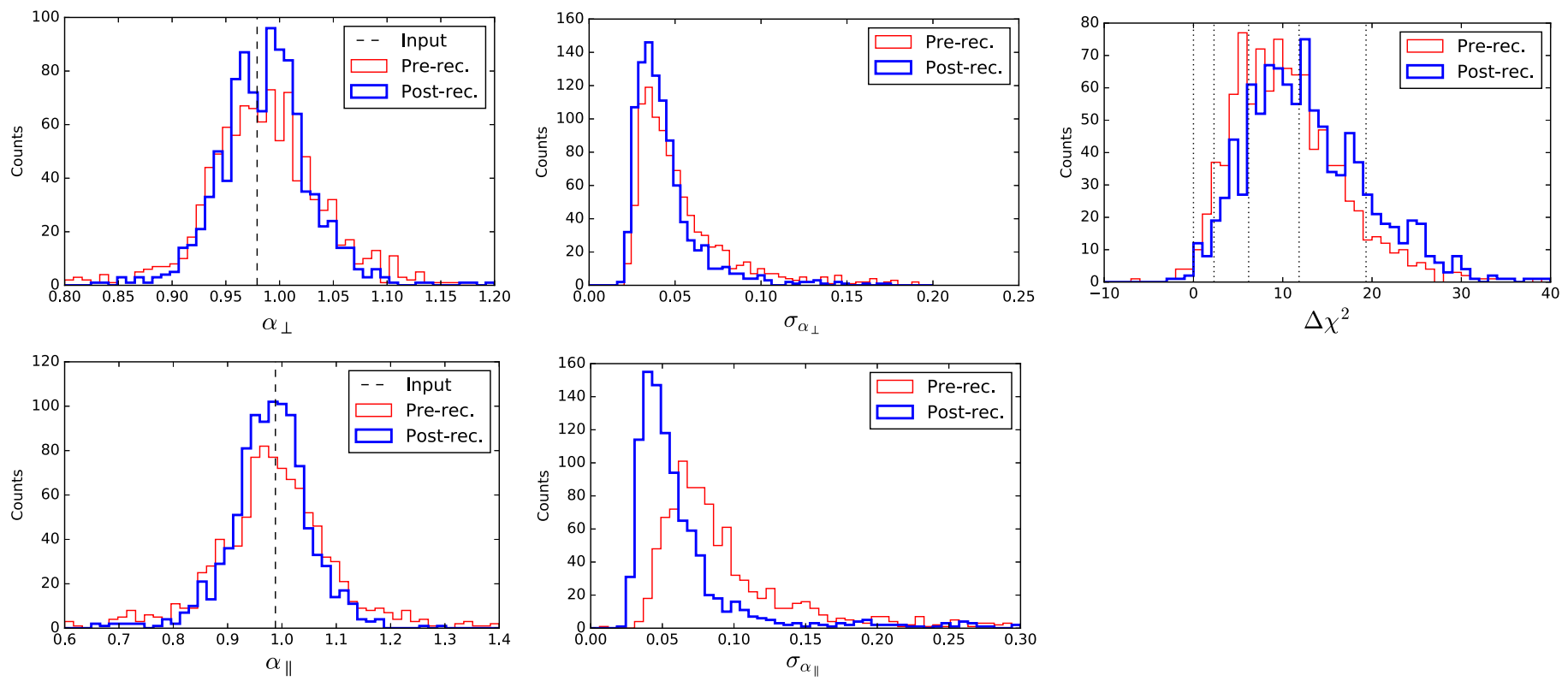

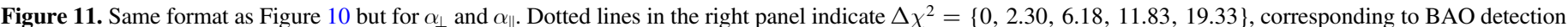
significances of $0,1,2,3$, and $4 \sigma$, respectively. The reduction of the width of the $\alpha$ histograms is also seen as a global reduction of $\sigma_{\alpha}$ in the middle panels.

Table 4

Results of Anisotropic Fits on the Full Set of 1000 Reconstructed Mock Catalogs

\begin{tabular}{|c|c|c|c|c|c|c|c|c|c|}
\hline Case & $N_{\text {good }}$ & $\left\langle\alpha_{\perp}-\alpha_{\perp 0}\right\rangle$ & $\operatorname{rms}\left(\alpha_{\perp}\right)$ & $\overline{\left\langle\sigma_{\alpha_{\perp}}\right\rangle}$ & $\overline{\left\langle\alpha_{\|}-\alpha_{\|_{0}}\right\rangle}$ & $\overline{\operatorname{rms}\left(\alpha_{\|}\right)}$ & $\left\langle\sigma_{\alpha_{\|}}\right\rangle$ & $\left\langle\chi_{r}^{2}\right\rangle$ & $\overline{\left\langle\Delta \chi^{2}\right\rangle}$ \\
\hline Prerec. & 887 & $0.0096 \pm 0.0021$ & 0.062 & 0.052 & $-0.0034 \pm 0.0038$ & 0.113 & 0.099 & 0.94 & 11.4 \\
\hline Postrec. (A) & 912 & $-0.0029 \pm 0.0014$ & 0.042 & 0.041 & $0.0499 \pm 0.0039$ & 0.116 & 0.103 & 0.95 & 11.6 \\
\hline Postrec. (B) & 900 & $0.0045 \pm 0.0015$ & 0.046 & 0.043 & $-0.0167 \pm 0.0035$ & 0.106 & 0.075 & 0.93 & 12.1 \\
\hline Postrec. (C) & 945 & $0.0086 \pm 0.0015$ & 0.046 & 0.043 & $-0.0131 \pm 0.0027$ & 0.084 & 0.064 & 0.94 & 13.2 \\
\hline
\end{tabular}

Note. Given the cosmological parameters in Table 1, the input values are $\alpha_{\perp 0}=0.9791$ and $\alpha_{\|_{0}}=0.9880$. All fits use $\Delta r=5 h^{-1}$ Mpc bins. The column $N_{\text {good }}$ shows the number of mocks where $\Delta \chi^{2}>2.3$ from which all numbers are computed.

Table 5

Isotropic Fits on Data for Different Choices of Binning

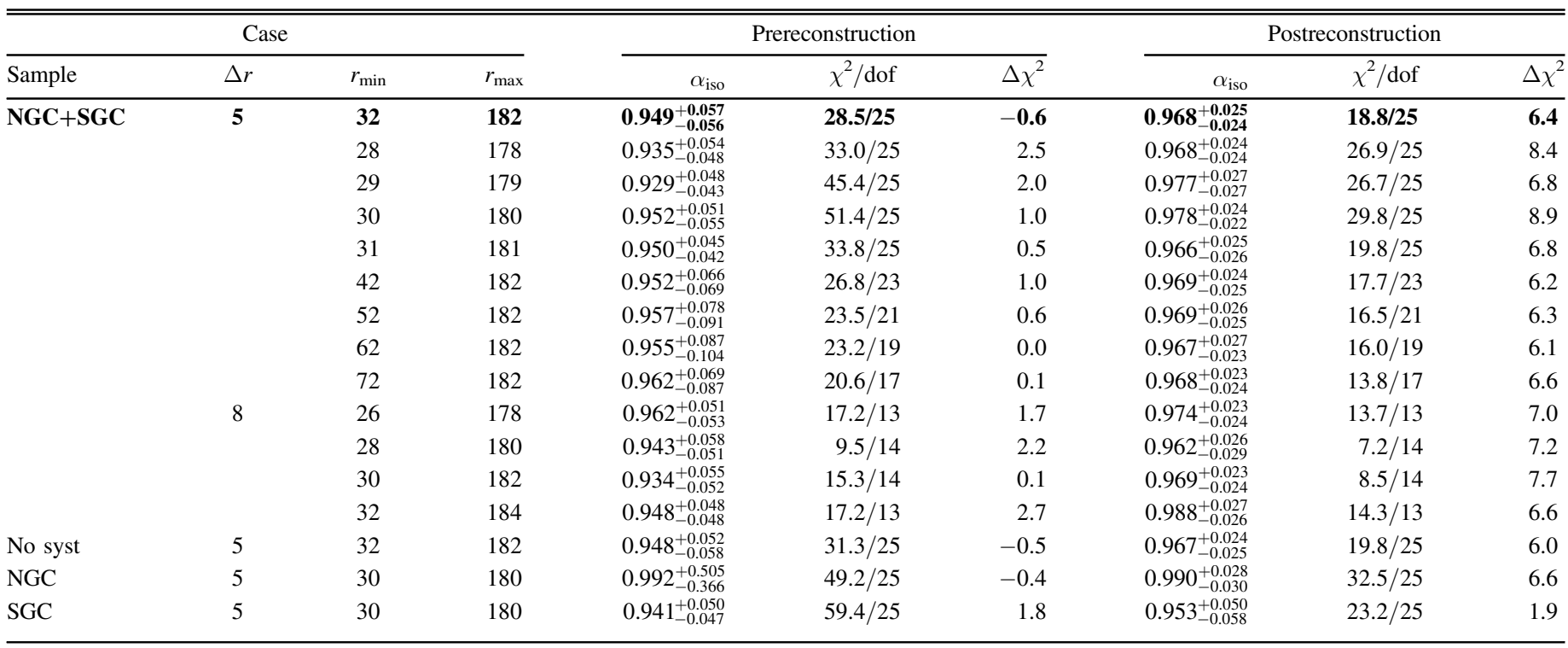

Note. In bold are the results for our fiducial analysis.

method A. On anisotropic fits, the bias on the pair $\left(\alpha_{\perp}, \alpha_{\|}\right)$ is also smaller for methods B and C. On average, method C produces more $\left(N_{\text {good }}\right)$ and better $\left(\left\langle\Delta \chi^{2}\right\rangle\right)$ BAO detections than the others. Therefore, we define method $\mathrm{C}$ as our fiducial reconstruction procedure to be applied on data. 
Table 6

Anisotropic Fits on Data for Different Binning Choices

\begin{tabular}{|c|c|c|c|c|c|c|c|c|c|c|c|c|}
\hline \multicolumn{3}{|c|}{ Case } & \multicolumn{5}{|c|}{ Prereconstruction } & \multicolumn{5}{|c|}{ Postreconstruction } \\
\hline$\overline{\Delta r}$ & $r_{\min }$ & $r_{\max }$ & $\alpha_{\perp}$ & $\overline{\alpha_{\|}}$ & $\chi^{2} / \mathrm{dof}$ & $\Delta \chi^{2}$ & $\overline{\text { corr. }}$ & $\alpha_{\perp}$ & $\overline{\alpha_{\|}}$ & $\chi^{2} /$ dof & $\Delta \chi^{2}$ & corr. \\
\hline \multirow[t]{6}{*}{5} & 32 & 182 & $1.00_{-0.06}^{+0.05}$ & $0.82_{-0.08}^{+0.09}$ & $75.0 / 50$ & 0.4 & -0.25 & $1.01_{-0.03}^{+0.06}$ & $\mathbf{0 . 8 8}_{-0.20}^{+0.06}$ & $66.2 / 50$ & 6.1 & -0.42 \\
\hline & 29 & 179 & $1.00_{-0.05}^{+0.08}$ & $0.83_{-0.06}^{+0.07}$ & $75.6 / 50$ & 3.5 & -0.47 & $1.03_{-0.04}^{+0.04}$ & $0.82_{-0.06}^{+0.08}$ & $54.3 / 50$ & 6.8 & -0.14 \\
\hline & 30 & 180 & $1.01_{-0.06}^{+0.12}$ & $0.82_{-0.08}^{+0.08}$ & $89.7 / 50$ & 1.9 & -0.47 & $1.02_{-0.03}^{+0.04}$ & $0.81_{-0.07}^{+0.12}$ & $61.5 / 50$ & 7.0 & -0.35 \\
\hline & 31 & 181 & $1.00_{-0.05}^{+0.06}$ & $0.83_{-0.08}^{+0.08}$ & $75.3 / 50$ & 2.5 & -0.30 & $1.00_{-0.04}^{+0.10}$ & $0.92_{-0.28}^{+0.06}$ & $62.6 / 50$ & 4.1 & -0.28 \\
\hline & 62 & 182 & $1.00_{-0.08}^{+0.13}$ & $0.82_{-0.07}^{+0.06}$ & $55.1 / 38$ & -1.2 & -0.39 & $1.00_{-0.03}^{+0.03}$ & $0.88_{-0.09}^{+0.33}$ & $56.1 / 38$ & -3.2 & -0.61 \\
\hline & 72 & 182 & $1.01_{-0.08}^{+0.11}$ & $0.82_{-0.07}^{+0.07}$ & $45.1 / 34$ & 2.4 & -0.37 & $1.00_{-0.03}^{+0.04}$ & $0.87_{-0.07}^{+0.08}$ & $37.0 / 34$ & 8.4 & -0.50 \\
\hline \multirow[t]{3}{*}{8} & 26 & 178 & $1.01_{-0.05}^{+0.08}$ & $0.82_{-0.08}^{+0.09}$ & $45.5 / 26$ & 2.8 & -0.39 & $1.02_{-0.04}^{+0.04}$ & $0.86_{-0.08}^{+0.08}$ & $35.6 / 26$ & 5.9 & -0.47 \\
\hline & 28 & 180 & $1.01_{-0.06}^{+0.11}$ & $0.81_{-0.08}^{+0.07}$ & $31.5 / 28$ & 3.0 & -0.68 & $1.00_{-0.04}^{+0.04}$ & $0.86_{-0.10}^{+0.06}$ & $25.9 / 28$ & 6.0 & -0.34 \\
\hline & 30 & 182 & $1.00_{-0.06}^{+0.13}$ & $0.82_{-0.07}^{+0.07}$ & $39.1 / 28$ & 1.5 & -0.69 & $1.00_{-0.03}^{+0.04}$ & $0.86_{-0.11}^{+0.07}$ & $30.1 / 28$ & 6.8 & -0.47 \\
\hline
\end{tabular}

Note. In bold are the results for our fiducial analysis (see text). Given the low statistical power of this sample for anisotropic fits, we do not include separate results for NGC or SGC.

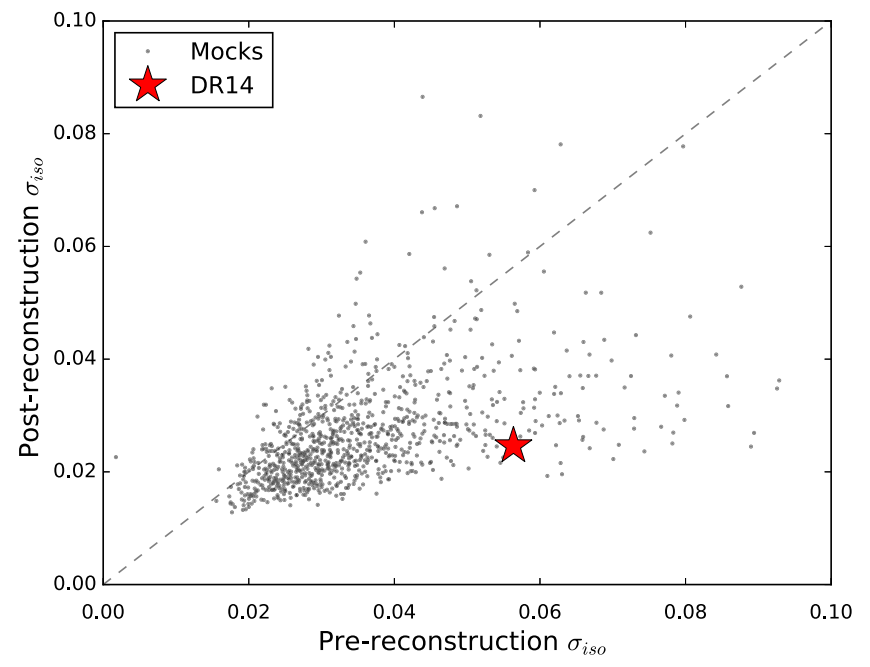

Figure 12. Estimated errors for the 1000 mocks. Prereconstruction results are shown in the $x$ axis, and the postreconstruction results (method C) are plotted on the $y$ axis. The red star indicates the result with our data sample. While the error prereconstruction with data is quite large compared to mocks, the error postreconstruction is typical of those found in mocks.

\section{Results}

In this section, we present our measurements and the basic tests performed on the robustness of those measurements.

\subsection{Fits to the Data}

We performed BAO fits on the final eBOSS+CMASS NGC + SGC sample (unless otherwise stated), using the covariance matrix derived from mock catalogs. We scale our covariance matrices by a factor of 0.9753 to account for the slight mismatch in footprint area between data and mocks. In order to test the robustness of our measurements, fits were performed with a set of different choices for binning, $\Delta r$, and separation ranges, $r_{\min }<r<r_{\max }$. We also tested the case where no correction of photometric systematics was computed. Our fiducial reconstruction method is method C (see Section 4.3).
Table 5 presents the results for isotropic fits pre- and postreconstruction. All $\alpha_{\text {iso }}$ values are stable and differ by less than $1 \sigma$. Estimated errors are also stable to the choice of analysis technique. The average significance for BAO detection in this sample is $\sim 1 . \sigma$ for prereconstruction, increasing to $\sim 2.6 \sigma$ after reconstruction. The estimated error on $\alpha_{\text {iso }}$ is reduced by $\sim 40 \%$ on average, and $\chi^{2}$ values become closer to the number of degrees of freedom after reconstruction. The observed reduction in errors represents a significant improvement when compared to mock catalogs. Figure 12 compares $\sigma_{\alpha_{\text {iso }}}$ obtained pre- and postreconstruction for mocks and data. While the data appear to be at the extremes of the distribution, the error postreconstruction is typical of that found in mocks.

Given the scatter in the significance of the measurement depending on the choice of analysis, we choose arbitrarily our fiducial analysis as the one producing the $\Delta \chi^{2}$ that is the closest to the average values of all cases in Table 5, that is, $\Delta r=$ $5 h^{-1} \mathrm{Mpc}$ and $\left(r_{\min }, r_{\max }\right)=(32,182) h^{-1}$ Mpc. We also adopt this choice of fiducial analysis in our anisotropic fits below.

Figure 13 shows the monopole and the two best-fit models (with and without BAO peak) on the reconstructed sample for our fiducial choice of analysis. Figure 14 presents the $\chi^{2}$ values as a function of $\alpha_{\text {iso }}$ and marginalizing over all other parameters for both models. The model without a peak has a $\chi_{\text {no peak }}^{2}$ about 8.0 units above the minimum of the model with peak, corresponding to a preference for the BAO peak model with a significance of $2.8 \sigma$.

Anisotropic fits are listed in Table 6 for the same analysis cases presented with isotropic fits. Best-fit $\alpha_{\perp}$ and $\alpha_{\|}$values are mostly stable to changes in the analysis. However, errors on the prereconstruction sample are quite unstable due to the low significance of the measurement $\left(\Delta \chi^{2} \sim 1.7\right.$ on average). Reconstruction slightly stabilizes errors and increases the significance of our constraints $\left(\Delta \chi^{2} \sim 5\right.$ on average). The estimated errors postreconstruction are consistent with the distribution of errors found in mock catalogs (see Table 4).

Figure 15 displays the best-fit anisotropic models compared to the data for our fiducial choice of analysis. We see how the 

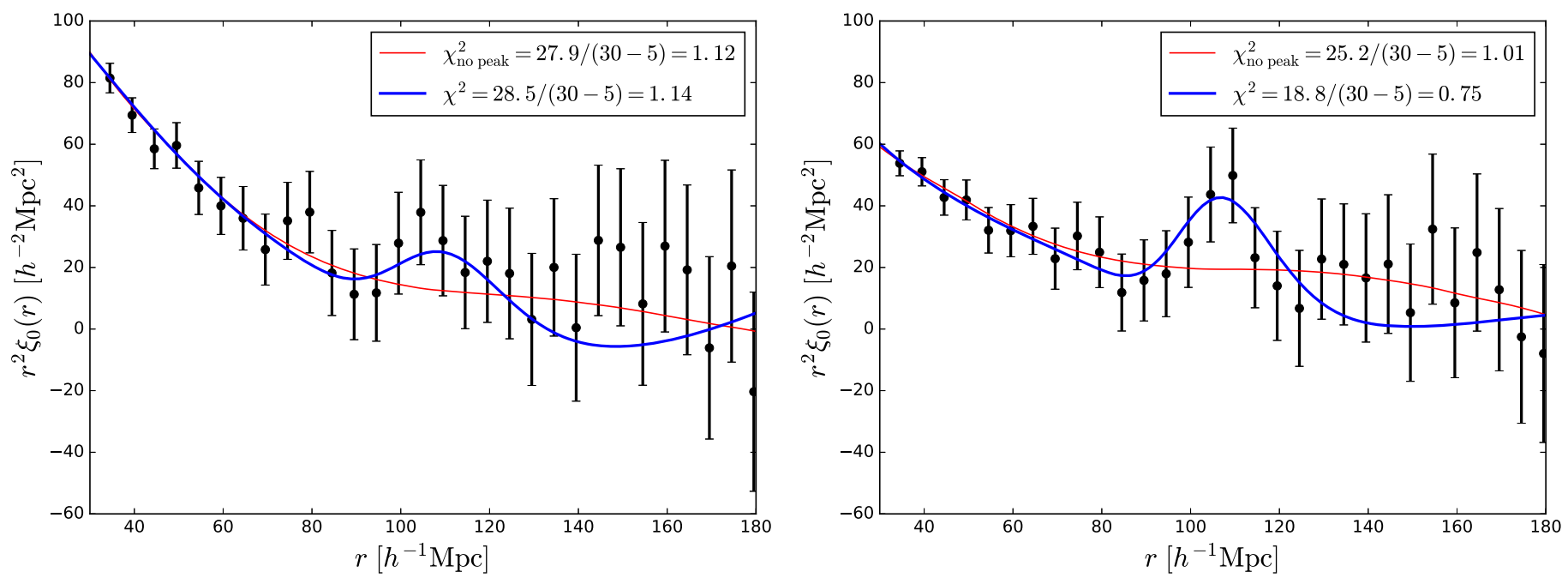

Figure 13. Monopole of the postreconstruction correlation function with the best-fit models with a BAO peak component (blue thick line) and without a peak (red thin line) for our fiducial choice of binning $\left(\Delta r=5 h^{-1} \mathrm{Mpc}\right.$ and $\left.32<r<182 h^{-1} \mathrm{Mpc}\right)$.

quadrupole is removed on intermediate scales thanks to reconstruction. Figure 16 shows the two-dimensional $\chi^{2}$ contours for $1 \sigma$ and $2 \sigma$, after converting $\alpha_{\perp}$ and $\alpha_{\|}$into $D_{M} / r_{d}$ and $D_{H} / r_{d}$, respectively, using values of our fiducial cosmological model on Equation (14). The likelihood becomes highly non-Gaussian beyond the $\Delta \chi^{2}=2.3$ contour due to the low statistical power of this sample. We expect that these contours will become more Gaussian as we increase the size of our data sample in the future.

Using our fiducial cosmology (Table 1), we convert our bestfit dilation parameters $\alpha_{\text {iso }}, \alpha_{\|}$, and $\alpha_{\perp}$ (bold postreconstruction values in Tables 5 and 6) into distance measurements. Our fiducial isotropic fit translates into

$$
D_{V}(z=0.72)=2377_{-59}^{+61}\left(r_{d} / r_{d, \text { fid }}\right) \mathrm{Mpc},
$$

while our anisotropic best-fit parameters yield

$$
\begin{aligned}
& D_{H}(z=0.72)=2593_{-589}^{+177}\left(r_{d} / r_{d, \text { fid }}\right) \mathrm{Mpc}, \\
& D_{M}(z=0.72)=2689_{-79}^{+158}\left(r_{d} / r_{d, \text { fid }}\right) \mathrm{Mpc} .
\end{aligned}
$$

\subsection{Comparison with Previous BAO Measurements}

We summarize current distance measurements using BAO in Figure 17. The distances are normalized to the predictions using a Planck cosmology. Our measurement of the isotropic BAO scale at $z=0.72$ is consistent with that of Planck at about the $1 \sigma$ level. The WiggleZ survey produced an isotropic BAO measurement at $z=0.73$ of $D_{V}(z=0.73)=2516 \pm 86\left(r_{d} / r_{d, \text { fid }}\right) \mathrm{Mpc}$ to be compared with Equation (19). These measurements show agreement within the $1 \sigma$ level. Both measurements are made over the same redshift range, $0.6<z<1.0$, but are nearly independent since the current overlap between eBOSS and WiggleZ is small (half of region "S1" of WiggleZ is covered by eBOSS LRGs). This overlap will be larger by the end of eBOSS observations, and a careful estimate of the correlations between the two surveys will be needed (as made with BOSS CMASS in Beutler et al. 2016).

Given that our eBOSS LRG sample was combined with the high-redshift tail of the CMASS sample in overlapping areas, the $\mathrm{BAO}$ measurements at $0.50<z<0.75$ from CMASS and ours, at $0.6<z<1.0$, are correlated. This correlation can be estimated by assuming that the covariance is proportional to the effective

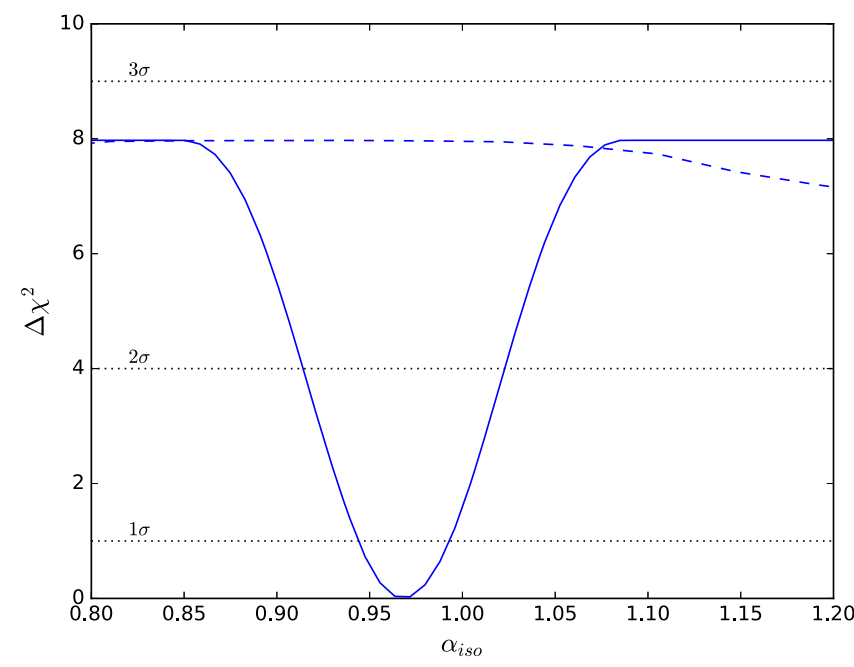

Figure 14. $\chi^{2}$ as a function of the dilation parameter $\alpha_{\text {iso }}$ for both models (solid: with peak; dashed: without peak). This curve corresponds to our fiducial choice of analysis $\left(\Delta s=5 h^{-1} \mathrm{Mpc}\right.$ and $\left.32<r<182 h^{-1} \mathrm{Mpc}\right)$. The $\Delta \chi^{2}=7.8$ corresponds to a detection of $2.8 \sigma$ of the BAO peak.

overlap volume between the two surveys. Using the CMASS $\bar{n}(z)$ over the effective area of eBOSS (overlapping area) covering $1844 \mathrm{deg}^{2}$, and computing Equation (5) over $0.6<$ $z<0.75$ (overlapping redshift range), we obtain an effective overlap volume of $V_{\text {eff }}\left[\right.$ CMASS $\cap$ eBOSS] $=0.31 \mathrm{Gpc}^{3}$. Therefore, we estimate the correlation coefficient between the two measurements to be

$$
\rho=\frac{V_{\text {eff }}(\text { CMASS } \cap \text { eBOSS })}{\sqrt{V_{\text {eff }}(\text { CMASS }) V_{\text {eff }}(\text { BOSS })}}=\frac{0.31}{\sqrt{4.1 \times 0.9}}=0.16 .
$$

We leave more realistic calculations of this correlation using correlated mock catalogs (as in, e.g., Beutler et al. 2016) for future work.

Forecasts in Zhao et al. (2016) predict $1 \%$ precision on isotropic BAO with $7000 \mathrm{deg}^{2}$ for the final eBOSS LRG sample (when combined with the high-redshift tail of CMASS). For the current footprint with $A_{\text {eff }}=1844 \mathrm{deg}^{2}$, the forecast scales to a $1.95 \% \mathrm{BAO}$ measurement assuming that the error is 

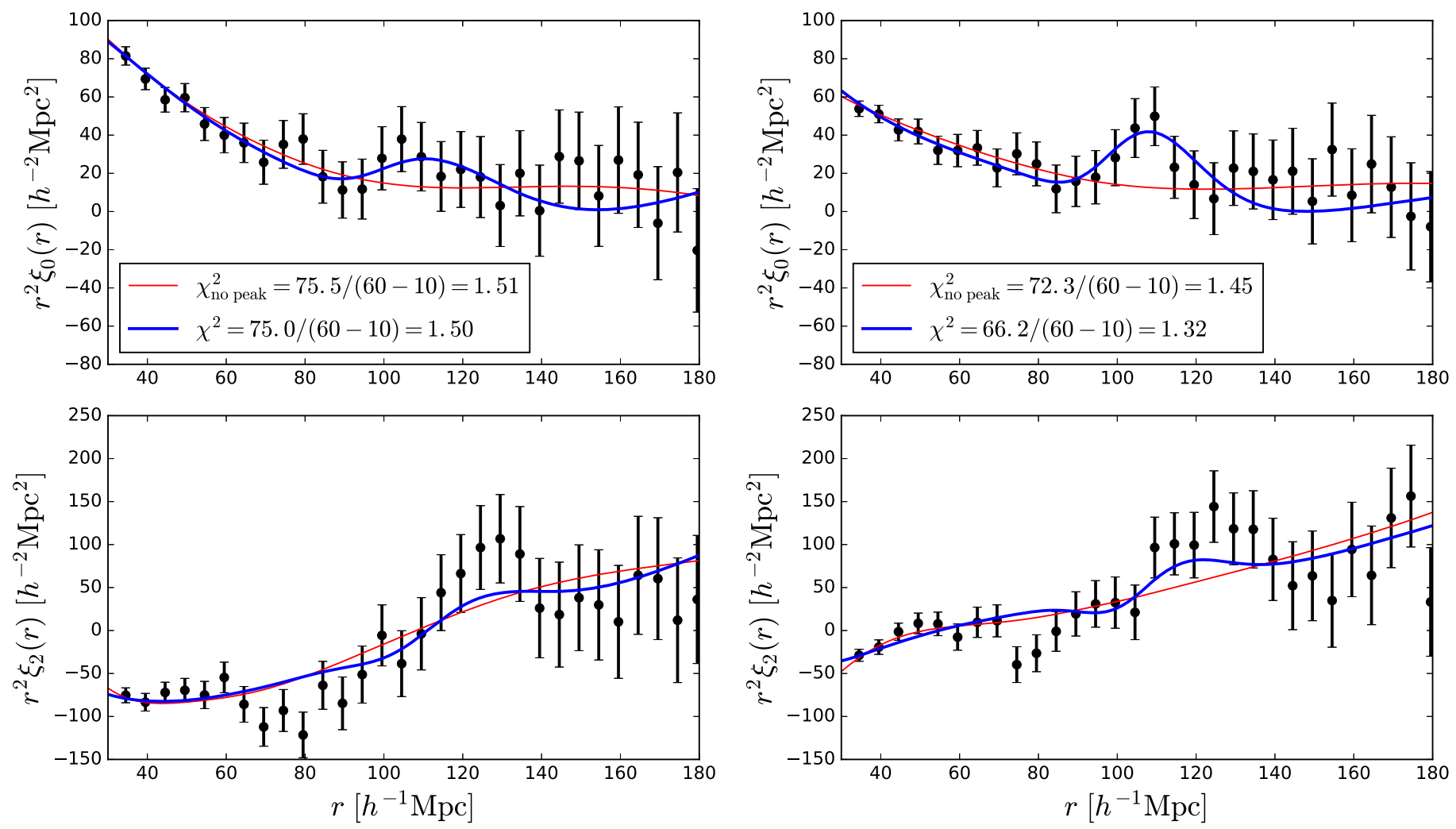

Figure 15. Monopole (top) and quadrupole (bottom) of the pre- (left) and postreconstruction (right) correlation function with their best-fit models with (blue thick line) and without (red thin line) a BAO peak component.

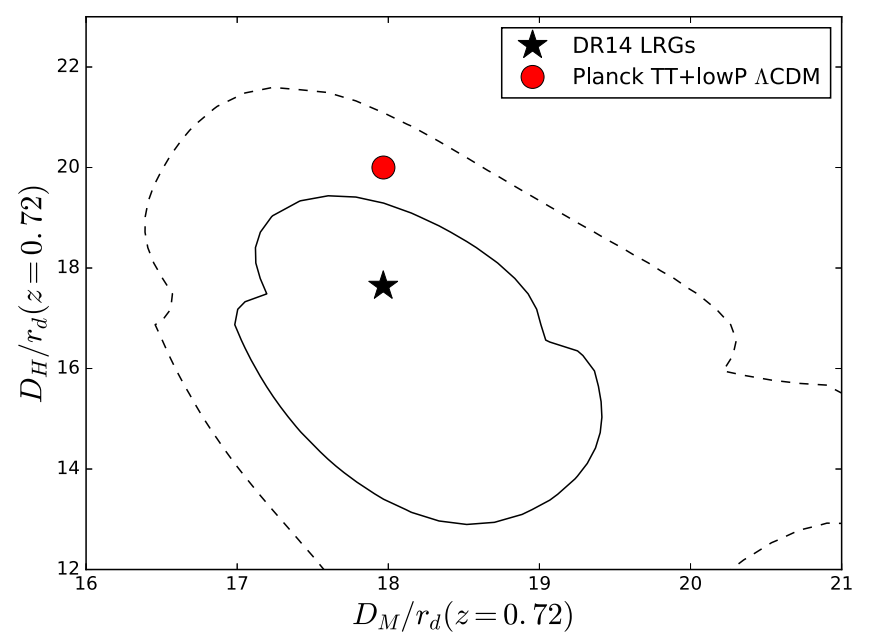

Figure 16. $\chi^{2}$ contours as a function of the dilation parameters $\alpha_{\perp}, \alpha_{\|}$ converted to $D_{M} / r_{d}$ and $D_{H} / r_{d}$ using our fiducial cosmology in Table 1 . The contours correspond to $\chi^{2}-\chi_{\min }^{2}=2.3,6.18,11.83$ (solid, dashed, and dotted, respectively).

proportional to the square root of the effective volume. Our isotropic BAO measurement with a $2.5 \%$ error is slightly larger than this forecast. This might be caused by holes in the footprint due to plates still not observed and to the various masks applied to our sample. These effects increase the size of boundaries of the survey and might increase errors relative to forecasts that consider uniform volumes. The larger error of our measurement compared to the forecast might also be due to statistical fluctuations, since the distribution of estimated errors

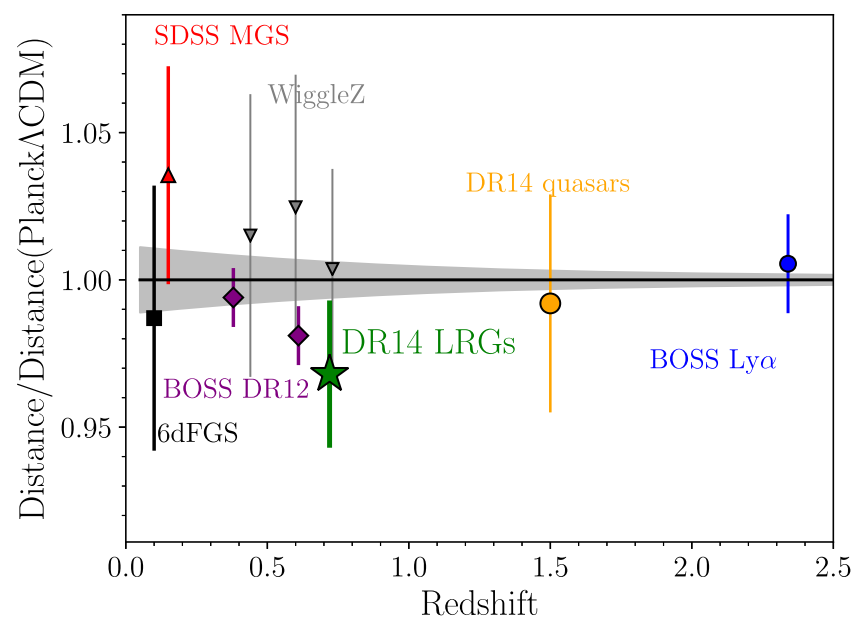

Figure 17. Current isotropic BAO measurements as a function of redshift compared to the prediction given by the best-fit cosmological parameters of Planck TT $+\mathrm{TE}+\mathrm{EE}+$ lowP (Planck Collaboration XIII et al. 2016). Our measurement is indicated by the green star labeled "DR14 LRGs." The other BAO measurements are 6dFGS at $z=0.11$ (Beutler et al. 2011), SDSS MGC at $z=0.15$ (Ross et al. 2015), BOSS DR12 at $z=[0.38,0.61]$ (Alam et al. 2017), WiggleZ at $z=[0.44,0.6$, 0.73] (Kazin et al. 2014), eBOSS DR14 QSO sample at $z=1.52$ (Ata et al. 2018), and BOSS DR12 Ly $\alpha$ sample at $z=2.3$ (Bautista et al. 2017; du Mas des Bourboux et al. 2017).

has a large dispersion, as observed with mock catalogs in Figure 10. Previous BAO measurements (e.g., Alam et al. 2017; Ata et al. 2018) typically also report estimated errors that are larger than predictions. 


\section{Conclusion}

We present the first $\mathrm{BAO}$ measurement using LRGs from the first two years of data taken in the eBOSS survey. The total area observed, weighted by the fiber completeness, is $1844 \mathrm{deg}^{2}$, yielding an effective volume of $0.9 \mathrm{Gpc}^{3}$ over $0.6<z<1.0$ when combining the eBOSS LRG sample with the CMASS $z>0.6$ galaxies over the eBOSS footprint. We obtain a $2.5 \%$ spherically averaged distance measurement after reconstruction at $z_{\text {eff }}=0.72$ that is consistent at the $1 \sigma$ level with the predictions of the $\Lambda \mathrm{CDM}$ model assuming a Planck best-fit cosmology.

In this analysis, we introduce a novel technique to account for redshift failures, while also propagating photometric systematics to the random catalog. This technique yields unbiased measurements of the correlation function, as tested on mock catalogs, and will be essential for future analyses using the full shape information such as redshift-space distortion studies.

When eBOSS will have finished its observing program, we expect that $7000 \mathrm{deg}^{2}$ of area will have been observed spectroscopically, representing a reduction on errors of isotropic BAO measurements of a factor of $\sqrt{7000 / 1844} \sim 2$ (assuming errors scale with the square root of the area).

The new software modules used to produce catalogs, compute the model for failures, fit the BAO peak, and apply reconstruction are all implemented in Python and available at github.com/julianbautista/eboss_clustering.

Upcoming surveys will significantly improve upon our results; the DESI survey will observe LRG spectra at larger density and area over the same redshift range, producing nearly an order of magnitude smaller errors on BAO. However, spectra will have $\mathrm{S} / \mathrm{N}$ similar to eBOSS, thus implying similar failure rates (if we neglect improvements in redshift determinations). Therefore, we expect that the framework presented here should be applicable for DESI clustering measurements using both LRGs and ELGs, where subpercent errors on BAO are expected.

This paper represents an effort by both the SDSS-III and SDSS-IV collaborations. Funding for SDSS-III was provided by the Alfred P. Sloan Foundation, the Participating Institutions, the National Science Foundation, and the U.S. Department of Energy Office of Science. Funding for the Sloan Digital Sky Survey IV has been provided by the Alfred P. Sloan Foundation, the U.S. Department of Energy Office of Science, and the Participating Institutions. SDSS-IV acknowledges support and resources from the Center for HighPerformance Computing at the University of Utah. The SDSS website is www.sdss.org.

SDSS-IV is managed by the Astrophysical Research Consortium for the Participating Institutions of the SDSS Collaboration including the Brazilian Participation Group, the Carnegie Institution for Science, Carnegie Mellon University, the Chilean Participation Group, the French Participation Group, Harvard-Smithsonian Center for Astrophysics, Instituto de Astrofísica de Canarias, The Johns Hopkins University, Kavli Institute for the Physics and Mathematics of the Universe (IPMU)/University of Tokyo, Lawrence Berkeley National Laboratory, Leibniz Institut für Astrophysik Potsdam (AIP), Max-Planck-Institut für Astronomie (MPIA Heidelberg), MaxPlanck-Institut für Astrophysik (MPA Garching), Max-PlanckInstitut für Extraterrestrische Physik (MPE), National
Astronomical Observatory of China, New Mexico State University, New York University, University of Notre Dame, Observatário Nacional/MCTI, The Ohio State University, Pennsylvania State University, Shanghai Astronomical Observatory, United Kingdom Participation Group, Universidad Nacional Autónoma de México, University of Arizona, University of Colorado Boulder, University of Portsmouth, University of Utah, University of Virginia, University of Washington, University of Wisconsin, Vanderbilt University, and Yale University.

This paper includes targets derived from the images of the Wide-Field Infrared Survey Explorer, which is a joint project of the University of California, Los Angeles, and the Jet Propulsion Laboratory/California Institute of Technology, funded by the National Aeronautics and Space Administration.

The support and resources from the Center for HighPerformance Computing at the University of Utah are gratefully acknowledged.

The work of J.B. and K.D. was supported in part by U.S. Department of Energy, Office of Science, Office of High Energy Physics, under award DE-SC0009959.

M.V. is partially supported by Programa de Apoyo a Proyectos de Investigación e Innovación Tecnológica (PAPITT) no. IA102516, Proyecto Conacyt Fronteras no. 281, and from Proyecto LANCAD-UNAM-DGTIC-319.

W.J.P. and E.-M.M. acknowledge support from the UK Science and Technology Facilities Council grant ST/ N000668/1. W.J.P. also acknowledges support from the European Research Council through the Darksurvey grant 614030 and the UK Space Agency grant ST/N00180X/1.

G.B.Z. is supported by NSFC grants 1171001024 and 11673025. G.B.Z. is also supported by a Royal Society Newton Advanced Fellowship, hosted by University of Portsmouth.

\section{ORCID iDs}

Julian E. Bautista (i https://orcid.org/0000-0002-9885-3989 Kyle S. Dawson (1) https://orcid.org/0000-0002-0553-3805 Joel Brownstein (i) https://orcid.org/0000-0002-8725-1069 Jeffrey A. Newman (1) https://orcid.org/0000-0001-8684-2222 Abhishek Prakash (1) https://orcid.org/0000-0003-4451-4444 Zhongxu Zhai $\odot$ https://orcid.org/0000-0001-7984-5476

\section{References}

Aihara, H., Allende Prieto, C., An, D., et al. 2011, ApJS, 193, 29 Alam, S., Albareti, F. D., Allende Prieto, C., et al. 2015, ApJS, 219, 12 Alam, S., Ata, M., Bailey, S., et al. 2017, MNRAS, 470, 2617 Albofathi, B., Aguado, D., Aguilar, G., et al. 2018, ApJS, 235, 42 Amendola, L., Appleby, S., \& Bacon, D. 2013, LLR, 16, 6 Anderson, L., Aubourg, E., Bailey, S., et al. 2012, MNRAS, 427, 3435 Anderson, L., Aubourg, E., Bailey, S., et al. 2014a, MNRAS, 439, 83 Anderson, L., Aubourg, E., Bailey, S., et al. 2014b, MNRAS, 441, 24 Ata, M., Baumgarten, F., Bautista, J., et al. 2018, MNRAS, 473, 4773 Bautista, J., Busca, N., Guy, J., et al. 2017, A\&A, 603, A12 Beutler, F., Blake, C., Colless, M., et al. 2011, MNRAS, 416, 3017 Beutler, F., Blake, C., Koda, J., et al. 2016, MNRAS, 455, 3230 Beutler, F., Seo, H.-J., Ross, A., et al. 2017, MNRAS, 464, 3409 Beutler, F., Shun, S., Brownstein, J., et al. 2014, MNRAS, 444, 3501 Bianchi, D., \& Percival, W. J. 2017, MNRAS, 472, 1106 Blake, C., Brough, S., Colless, M., et al. 2010, MNRAS, 406, 803 Blake, C., Kazin, E. A., Beutler, F., et al. 2011, MNRAS, 418, 1707 Blanton, M., \& Bershady, M. 2017, AJ, 154, 28

Bolton, A., Schlegel, D. J., Aubourg, E., et al. 2012, AJ, 144, 144 Bond, R., \& Efstathiou, G. 1987, MNRAS, 226, 665

Burden, A., Percival, W. J., \& Howlett, C. 2015, MNRAS, 453, 456 Burden, A., Percival, W. J., \& Manera, M. 2014, MNRAS, 445, 3152 
Clifton, T., Ferreira, P., Padilla, A., \& Skordis, C. 2012, PhR, 513, 1 Cole, S., Percival, W. J., Peacock, J. A., et al. 2005, MNRAS, 362, 505 Colless, M., Peterson, B. A., Jackson, C., et al. 2003, arXiv:astro-ph/0306581 Cui, W., Liu, L., Yang, X., et al. 2008, ApJ, 687, 738

Cunha, E., Hopkins, A., Colless, M., et al. 2017, PASP, 34, e047

Dawson, K. S., Kneib, J.-P., Percival, W. J., et al. 2016, AJ, 151, 44

Dawson, K. S., Schlegel, D. J., Ahn, C. P., et al. 2013, AJ, 145, 10 de Jong, R. S., Barden, S., Bellido-Tirado, O., et al. 2016, Proc. SPIE, 9908, 1 Delubac, T., Raichoor, A., Comparat, J., et al. 2017, MNRAS, 465, 1831 DESI Collaboration 2016a, arXiv:1611.00036 DESI Collaboration 2016b, arXiv:1611.00037

Doi, M., Tanaka, M., Fukugita, M., et al. 2010, AJ, 139, 1628

Drinkwater, M., Russell, J., Blake, C., et al. 2010, MNRAS, 401, 1429

du Mas des Bourboux, H., le Goff, J. M., Blomqvist, M., et al. 2017, A\&A, 608,130

Eisenstein, D. J., Seo, H.-J., Sirko, E., \& Spergel, D. N. 2007, ApJ, 664, 675 Eisenstein, D. J., Weinberg, D. H., Agol, E., et al. 2011, AJ, 142, 72 Eisenstein, D. J., Zehavi, I., Hogg, D. W., et al. 2005, ApJ, 633, 560 Feldman, H. A., Kaiser, N., \& Peacock, J. A. 1994, ApJ, 426, 23 Font-Ribera, A., McDonald, P., Mostek, N., et al. 2014, JCAP, 05, 23 Fukugita, M., Ichikawa, T., Gunn, J., et al. 1996, AJ, 111, 1748 Gil-Marín, H., Percival, W., Verde, L., et al. 2017, MNRAS, 465, 1757

Gunn, J. E., Siegmund, W. A., Mannery, E. J., et al. 2006, AJ, 131, 2332 Guo, H., Zehavi, I., \& Zheng, Z. 2012, ApJ, 756, 2

Hartlap, J., Simon, P., \& Schneider, P. 2007, A\&A, 464, 399

Ho, S., Cuesta, A., Seo, H.-J., et al. 2012, ApJ, 761, 14

Høg, E., Fabricius, C., Makarov, V. V., et al. 2000, A\&A, 355, 27

Hutchinson, T., Bolton, A., \& Dawson, K. 2016, AJ, 152, 205

Jensen, T., Vivek, M., \& Dawson, K. 2016, ApJ, 833, 199

Jones, D. H., Read, M., Saunders, W., et al. 2009, MNRAS, 339, 683

Kazin, E., Koda, J., Blake, C., et al. 2014, MNRAS, 441, 3524

Kirkby, D., Margala, D., Slosar, A., et al. 2013, JCAP, 3, 24

Landy, S. D., \& Szalay, A. S. 1993, ApJ, 412, 64
Lang, D., Hogg, D. W., \& Schlegel, D. J. 2014, arXiv:1410.7397

Laureijs, R., Amiaux, J., Arduini, S., et al. 2011, arXiv:1110.3193

Lewis, A., Challinor, A., \& Lasenby, A. 2016, ApJ, 538, 473

Myers, A., Palanque-Delabrouille, N., Prakash, A., et al. 2015, ApJS, 221, 27

Peebles, P., \& Yu, J. 1970, ApJ, 162, 815

Percival, W. J. 2001, MNRAS, 327, 1297

Percival, W. J., Cole, S., Eisenstein, D. J., et al. 2007, MNRAS, 381, 1053

Percival, W. J., Ross, A. J., Sánchez, A. G., et al. 2014, MNRAS, 439, 2531

Planck Collaboration I, Ade, P. A. R., et al. 2016, A\&A, 594, 1

Planck Collaboration XIII, Ade, P. A. R., et al. 2016, A\&A, 594, A13

Prakash, A., Licquia, T., Newman, J., \& Rao, S. M. 2015, ApJ, 803, 105

Prakash, A., Licquia, T., Newman, J., et al. 2016, ApJS, 224, 34

Raichoor, A., Comparat, J., Delubac, T., et al. 2017, MNRAS, 471, 3955

Reid, B., Ho, S., Padmanabhan, N., et al. 2016, MNRAS, 455, 1553

Ross, A., Beutler, F., Chuang, C.-H., et al. 2017, MNRAS, 464, 1668

Ross, A., Ho, S., Cuesta, A., et al. 2011, MNRAS, 417, 1350

Ross, A., Percival, W., Sánchez, A., et al. 2012, MNRAS, 424, 564

Ross, A. J., Samushia, L., Howlett, C., et al. 2015, MNRAS, 449, 835

Rykoff, E. S., Rozo, E., Busha, M. T., et al. 2014, ApJ, 785, 104 SDSS Collaboration 2016, arXiv:1608.02013

Seo, H.-J., Beutler, F., Ross, A., \& Saito, S. 2016, MNRAS, 460, 2453

Smee, S., Gunn, J. E., Uomoto, A., et al. 2013, AJ, 146, 32

Sunyaev, R., \& Zeldovich, Y. 1970, Ap\&SS, 7, 3

Taylor, A., Joachimi, B., \& Kitching, T. 2014, MNRAS, 432, 1928

Tojeiro, R., Ross, A. J., Burden, A., et al. 2014, MNRAS, 440, 2222

Vargas-Magaña, M., Ho, S., Fromenteau, S., et al. 2017, MNRAS, 467, 2331

Weinberg, D., Mortonson, M., Eisenstein, D., et al. 2013, PhR, 530, 87

White, M. 2015, MNRAS, 450, 3822

White, M., Tinker, J. L., \& McBride, C. K. 2014, MNRAS, 437, 2594

Wright, E., Eisenhardt, P., \& Mainzer, A. 2010, AJ, 140, 1868

York, D. G., Adelman, J., Anderson, J. E., et al. 2000, AJ, 120, 1579

Zhai, Z., Tinker, J., Hahn, C., et al. 2017, ApJ, 848, 76

Zhao, G., Wang, Y., Ross, A., et al. 2016, MNRAS, 457, 2377 